\title{
Analysis of Linkage for Ten X-STR Markers in a Rio de Janeiro (Brazil) Three-Generation Family Sample
}

\author{
Roberto Chan1, Elizeu Fagundes de Carvalho², Juliana Gozi de Aquino², \\ Dayse Aparecida da Silva ${ }^{2}$, Gisele Lôbo-Hajdu ${ }^{3}$ \\ ${ }^{1}$ Coordenação de Astronomia e Astrofísica, Observatório Nacional, Rio de Janeiro, Brazil \\ ${ }^{2}$ DNA Diagnostic Laboratory (LDD), State University of Rio de Janeiro (UERJ), Rio de Janeiro, Brazil \\ ${ }^{3}$ Department of Genetics, State University of Rio de Janeiro (UERJ), Rio de Janeiro, Brazil \\ Email: chan@on.br, elizeufc@hotmail.com, dayse.a.silva@gmail.com, glhajdu@uerj.br
}

Received 6 April 2014; revised 5 May 2014; accepted 4 June 2014

Copyright (C) 2014 by authors and Scientific Research Publishing Inc.

This work is licensed under the Creative Commons Attribution International License (CC BY).

http://creativecommons.org/licenses/by/4.0/

(c) (i) Open Access

\begin{abstract}
Recently, typing of polymorphisms on the $\mathrm{X}$ chromosome has become a standard technique in forensic genetics and a growing number of short tandem repeats (STR) has been established in this chromosome related to genetic population studies. Knowledge of marker recombination is very important especially when the $X$ chromosome typing is used in forensic kinship analysis. It is known that the meiotic recombination is not a simple function of the physical distance between segments of the DNA but the recombination events between them tend to be clustered at special regions of the chromosome. Information on the rate of recombination among markers can be gathered by studying families through several generations. In this work we have typed DNA samples of pedigree consisting of nineteen families in Rio de Janeiro, constituted of grandfather, mother and grandson, and in some cases grandmother and aunt, and reported the recombination of 10 STR markers of the $X$ chromosome. The study of the linkage analysis using the LOD score has shown that the marker pairs DXS8378-DXS7423, DXS7132-DXS9898, DXS7132-GATA172D05 DXS9898-DXS7133 and DXS6809-DXS7133 are not transmitted in a random way, during a recombination event.
\end{abstract}

\section{Keywords}

X-Chromosome STR Markers, Recombination, Probability of Linkage, LOD Score

\section{Introduction}

The benchmark of using the $\mathrm{X}$ chromosome markers in the forensic practice can be found in the clinical genetics

How to cite this paper: Chan, R., de Carvalho, E.F., de Aquino, J.G., da Silva, D.A. and Lôbo-Hajdu, G. (2014) Analysis of Linkage for Ten X-STR Markers in a Rio de Janeiro (Brazil) Three-Generation Family Sample. Open Journal of Genetics, 4, 245285. http://dx.doi.org/10.4236/ojgen.2014.43025 
around the years 1950. The haemophilia and the red-green blindness, for example, are diseases caused by the recessive genes linked to the $\mathrm{X}$ chromosome. As the women inherit two $\mathrm{X}$ chromosomes, they may be homozygote for the recessive allele, therefore the allele related to the disease is doubled, heterozygote or homozygote for the normal and dominant alleles at this locus. In women the disease happens when they have recessive alleles for the disease gene in homozygose, while in men, due to the $\mathrm{X}$ chromosome hemyzigose, happens with only one recessive allele at this locus [1].

If the disease carrier is a fertile male patient, his daughters will always inherit the defectfull $\mathrm{X}$ chromosome. Consequently, the daughters will transmit with $50 \%$ of probability to next generation. Such paternal heritage pattern of the $\mathrm{X}$ chromosome to his daughters has become an additional method of using the $\mathrm{X}$ chromosome markers in the genetic kinship analysis.

Besides, if a man has two or more $\mathrm{X}$ chromosome alleles linked physically it is obvious that these alleles must be unified in a haplotype. In the context of forensic analysis, the challenge is the stablishment of methods that can bypass the relative deficiency in the kinship testings. A possibility is to use $\mathrm{X}$ chromosome markers haplotypes. The method of substituting the individual STR markers by haplotypes can furnish useful tools when it is applied to kinship testing for individuals of the female gender. This is the reason of the importance of the analysis of linkage of X-STR markers using the LOD score [2].

The Brazilian population was formed by successive migratory waves. The Amerindian people were occupying the Brazilian territory when the Portuguese arrived in 1500, and colonized the country. Between the 16th and the 19th centuries Africans were brought to Brazil as slaves, and besides the Portuguese, other migratory waves occurred in the 19th and 20th centuries, mainly from Italy, Germany and Spain. All these migratory events have contributed to the formation of a multiethnic highly mixed population. This heterogeneity is due to the nonuniform triethnic (European + African + Amerindian) pattern for the Brazilian population gene pool [3].

\section{Materials and Methods}

A population sample of 19 families, whose individuals are unrelated from each other, was selected from the population of the Brazilian state Rio de Janeiro with 45 males and 23 females.

\subsection{X-STR Amplification}

For the PCR amplification of the target loci we used pairs of primers in a decaplex system [4], the QIAGEN Multiplex PCR Kit, at $1 \times$ QIAGEN multiplex PCR master mix, mix of primers at concentration of $0.2 \mu \mathrm{M}$ and 1 - 2 ng of DNA, in a final volume of 6.5 to $10 \mu \mathrm{L}$. Primers were fluorescent labeled with 6-FAM (DXS8378, DXS9898, DXS7133, GATA31E08), with VIC (GATA172D05, DXS7423, DXS6809) and with NED (DXS 7132, DXS9902, DXS6789) (see Table 1, Table 2 and Figure 1).

Thermocycling conditions were: pre-incubation for $15 \mathrm{~min}$ at $95^{\circ} \mathrm{C}$, followed by ten cycles of $30 \mathrm{~s}$ at $94^{\circ} \mathrm{C}, 90$ s at $60^{\circ} \mathrm{C}, 60 \mathrm{~s}$ at $72^{\circ} \mathrm{C}$; and 20 cycles of $30 \mathrm{~s}$ at $94^{\circ} \mathrm{C}, 90 \mathrm{~s}$ at $58^{\circ} \mathrm{C}$, and $60 \mathrm{~s}$ at $72^{\circ} \mathrm{C}$ with a final incubation for $60 \mathrm{~min}$ at $72^{\circ} \mathrm{C}[4]$.

\subsection{Analysis of PCR Products}

Aliquots of $1 \mathrm{~mL}$ of PCR product were mixed with $8.8 \mathrm{~mL}$ formamide and $0.2 \mathrm{~mL}$ of ILS 500 size standard (Applied Biosystem) and separated by capillary electrophoresis on an ABI Prism 3130 Avant Genetic Analyzer instrument with denaturing polymer 3100 POP-7TM (Performance Optimized Polymer-Applied Biosystems) with GeneMapper v 3.01 Analysis Software (Applied Biosystems). In the Appendix A we present all the XSTR typing of all the families.

In order to determine if two loci are linked we must use the following procedure: (a) Typing the $\mathrm{X}$ chromosome markers of the families with grandfather, mother and grandson; (b) Counting the number of children that shows or does not show recombination between these two loci; (c) Calculating the LOD score using the equation (1); (d) Maximizing the Equation (1) in order to find $\theta_{\max }$; (e) Finally, obtaining $Z_{\max }=Z\left(\theta_{\max }\right)$. The LOD score is given by

$$
Z=\log _{10}\left[\frac{\theta^{m}(1-\theta)^{n}}{(1 / 2)^{m}(1 / 2)^{n}}\right]=\log _{10}\left[2^{m} 2^{n} \theta^{m}(1-\theta)^{n}\right]
$$


Table 1. Sequence of the primers of the decaplex system [4].

\begin{tabular}{|c|c|}
\hline LOCUS & PRIMERS (5’ - 3’) \\
\hline \multirow[t]{2}{*}{ DXS8378 } & CACAGGAGGTTTGACCTGTT \\
\hline & AACTGAGATGGTGCCACTGA \\
\hline \multirow[t]{2}{*}{ DXS9902 } & TGGAGTCTCTGGGTGAAGAG \\
\hline & CAGGAGTATGGGATCACCAG \\
\hline \multirow[t]{2}{*}{ DXS7132 } & TGATTAGGAATATCAAAGGCAAA \\
\hline & СTTCTCTGGTTCTCTAGCTCACAT \\
\hline \multirow[t]{2}{*}{ DXS9898 } & CGAGCACACCTACAAAAGCT \\
\hline & TCGATTAGGTTCAGTTCCCA \\
\hline \multirow[t]{2}{*}{ DXS6809 } & TGAACCTTCCTAGCTCAGGA \\
\hline & TCTGGAGAATCCAATTTTGC \\
\hline \multirow[t]{2}{*}{ DXS6789 } & TTGGTACTTAATAAACCСТСТTTT \\
\hline & CTAGAGGGACAGAACCAATAGG \\
\hline \multirow[t]{2}{*}{ DXS7133 } & GCTTCCTTAGATGGCATTCA \\
\hline & CTTCCAAGAATCAGAAGTCTCC \\
\hline \multirow[t]{2}{*}{ GATA172D05 } & TAGTGGTGATGGTTGCACAG \\
\hline & ATAATTGAAAGCCCGGATTC \\
\hline \multirow[t]{2}{*}{ GATA31E08 } & AGGGGAGAAGGCTAGAATGA \\
\hline & CAGCTGACAGAGCACAGAGA \\
\hline \multirow[t]{2}{*}{ DXS7423 } & GTCTTCСТGTCATCTCССААС \\
\hline & TAGCTTAGCGCCTGGCACATA \\
\hline
\end{tabular}

Table 2. The identification locus number.

\begin{tabular}{ccc}
\hline$\#$ & Locus & Xp-tel Distance \\
\hline 1 & DXS8378 & 9330 \\
2 & DXS9902 & 15233 \\
3 & DXS7132 & 64572 \\
4 & DXS9898 & 87682 \\
5 & DXS6809 & 90180 \\
6 & DXS6789 & 95336 \\
7 & DXS7133 & 108928 \\
8 & GATA172D05 & 113061 \\
9 & GATA31E08 & 140800 \\
10 & DXS7423 & 149461 \\
\hline
\end{tabular}

Note: The distances from Xp-telomere (Xp-tel) are in unites of 1000 base pairs.

where $\theta$ is the recombination frequency, $m$ is the number of meiosis when recombination happens e $n$ is the number of meiosis when recombination between the two loci does not happen [5]. In the Appendix B we present the detailed linkage analysis for all the families. 


\section{Results and Discussion}

The study of the linkage analysis between two loci using the LOD score has shown (see Table 3) that the marker pairs DXS8378-DXS7423, DXS7132-DXS9898, DXS7132-GATA172D05, DXS9898-DXS7133 and DXS6809DXS7133 are linked during a crossing over event. Their LOD scores are greater or equal to three, i.e., they are at least a thousand times greater than the probability of a transmition of the alleles in a random way.

Table 3. The total maximum LOD scores are listed. The total is the sum of the LOD of the 19 families. Significant scores $(\geq 3)$ are denoted by the symbol.

\begin{tabular}{|c|c|}
\hline Combination of Markers & $Z_{\max }^{(\text {total })}$ \\
\hline DXS8378-DXS9902 & 2.54 \\
\hline DXS8378-DXS7132 & 2.70 \\
\hline DXS8378-DXS9898 & 2.17 \\
\hline DXS8378-DXS6809 & 2.10 \\
\hline DXS8378-DXS6789 & 1.80 \\
\hline DXS8378-DXS7133 & 2.40 \\
\hline DXS8378-GATA172D05 & 2.40 \\
\hline DXS8378-GATA31E08 & 2.70 \\
\hline DXS8378-DXS7423 & *3.30 \\
\hline DXS9902-DXS7132 & 1.80 \\
\hline DXS9902-DXS9898 & 1.80 \\
\hline DXS9902-DXS6809 & 2.40 \\
\hline DXS9902-DXS6789 & 1.87 \\
\hline DXS9902-DXS7133 & 2.17 \\
\hline DXS9902-GATA172D05 & 2.10 \\
\hline DXS9902-GATA31E08 & 2.47 \\
\hline DXS9902-DXS7423 & 2.17 \\
\hline DXS7132-DXS9898 & *3.00 \\
\hline DXS7132-DXS6809 & 2.70 \\
\hline DXS7132-DXS6789 & 2.70 \\
\hline DXS7132-DXS7133 & 2.40 \\
\hline DXS7132-GATA172D05 & *3.00 \\
\hline DXS7132-GATA31E08 & 2.47 \\
\hline DXS7132-DXS7423 & 2.77 \\
\hline DXS9898-DXS6809 & 2.40 \\
\hline DXS9898-DXS6789 & 2.70 \\
\hline DXS9898-DXS7133 & *3.00 \\
\hline DXS9898-GATA172D05 & 2.70 \\
\hline DXS9898-GATA31E08 & 1.87 \\
\hline DXS9898-DXS7423 & 2.17 \\
\hline DXS6809-DXS6789 & 2.40 \\
\hline DXS6809-DXS7133 & *3.00 \\
\hline DXS6809-GATA172D05 & 2.40 \\
\hline DXS6809-GATA31E08 & 2.10 \\
\hline DXS6809-DXS7423 & 2.40 \\
\hline DXS6789-DXS7133 & 1.80 \\
\hline DXS6789-GATA172D05 & 2.40 \\
\hline DXS6789-GATA31E08 & 1.80 \\
\hline DXS6789-DXS7423 & 2.10 \\
\hline DXS7133-GATA172D05 & 2.10 \\
\hline DXS7133-GATA31E08 & 1.80 \\
\hline DXS7133-DXS7423 & 2.10 \\
\hline GATA172D05-GATA31E08 & 2.40 \\
\hline GATA172D05-DXS7423 & 2.70 \\
\hline
\end{tabular}


BLOOD OR SWAB

SAMPLES

OF THE GP, M, GS
DNA EXTRACTION BY SALTING-OUT METHOD

DETERMINATION OF

CONCENTRATION OF

2 ng OF DNA USING

ESPECTROPHOTOMETRY<smiles>[3H][13CH3]</smiles>

STR AMPLIFICATION

USING DECAPLEX

SYSTEM [3]

ALLELES OBTAINED
USING THE ABI
ANALYSIS SOFTWARE
LINKAGE OF THE
ALLELES USING
LOD SCO RE I4]
$\begin{aligned} & \text { Figure 1. Flow chart depicting the process of obtaining the STR alleles of the X chro- } \\ & \text { mosome and the linkage analysis between them. }\end{aligned}$

In a recent work Hering and collaborators [2], using 3-generation families and $39 \mathrm{X}$-STRs, have studied four clusters of closely localized marker linkage groups: (I) DXS10148-DXS8378 at Xp21, (II) DXS7132-DXS981 at Xq12, (III) DXS10103-DXS10101 at Xq26 and (IV) DXS10146-DXS10011 at Xq28. They have found that there was independent segregation between linkage groups I/II and II/III. The genetic distance between groups III and IV was found to be too small to assume independence. Notice that in this work we have only two coincident markers (DXS8378 and DXS7132) and they are located in the linkage groups with independent segregation.

Finally, this work is the first one in the literature that shows this kind of linkage analysis for recombination in X-chromosome STRs, using a sample of families from Rio de Janeiro (Brazil).

\section{Acknowledgments}

We thank the financial support granted by Fundação Carlos Chagas Filho de Amparo à Pesquisa do Estado do Rio de Janeiro (FAPERJ), DNA Program - State University and Justice Court of Rio de Janeiro, Brazil and R.C. thanks also Conselho Nacional de Desenvolvimento Científico e Tecnológico (CNPq) Brazil.

\section{References}

[1] Szibor, R. (2007) X-Chromosomal Markers: Past, Present and Future. Forensic Science International: Genetics, 1, 9399. http://dx.doi.org/10.1016/j.fsigen.2007.03.003

[2] Hering, S., Edelmann, J., Augustin, C., Kuhlish, E. and Szibor, R. (2010) X Chromosomal Recombination-A Family Study Analysing 39 STR Markers in German Three-Generation Pedigrees. International Journal of Legal Medicine, 124, 483-491. http://dx.doi.org/10.1007/s00414-009-0387-y

[3] Leite, F.P.N., Santos, S.E.B., Rodríguez, E.M.R., Callegari-Jacques, S.M., Demarchi, D.A., Tsuneto, L.T., Petzl-Erler, M.L., Salzano, F.S. and Hutz, M.H. (2009) Linkage Disequilibrium Patterns and Genetic Structure of Amerindian and Non-Amerindian Brazilian Populations Revealed by Long-Range X-STR Markers. American Journal of Physical Antroppology, 139, 404-412. http://dx.doi.org/10.1002/ajpa.20998

[4] Gusmão, L., Sánchez-Diz, P., Alves, C., Gomes, I., Zarrabeitia, M.T., Abovich, M., Atmetlla, I., Bobillo, C., Bravo, L., Builes, J., Cainé, L., Calvo, R., Carvalho, E., Carvalho, M., Cicarelli, R., Catelli, L., Corach, D., Espinoza, M., García, O., Malaghini, M., Martins, J., Pinheiro, F., Porto, M.J., Raimondi, E., Riancho, J.A., Rodríguez, A., Rodríguez, A., Cardozo, B.R., Schneider, V., Silva, S., Tavares, C., Toscanini, U., Vullo, C., Whittle, M., Yurrebaso, I., Carracedo, A. and Amorim, A. (2009) A GEP-ISFG Collaborative Study on the Optimization of an X-STR Decaplex: Data on 15 
Iberian and Latin American Populations. International Journal of Legal Medicine, 123, 227-234. http://dx.doi.org/10.1007/s00414-008-0309-4

[5] Nussbaum, R.L., McInnes, R.R. and Willard, H.F. (2007) Thompson \& Thompson Genetics in Medicine. Elsevier Academy Press, London. 


\section{Appendix A}

In this appendix we present the Table 4 with the alleles of each member of the families.

Table 4. Typing of the $\mathrm{X}$ chromosome of families 1 to 19.

\begin{tabular}{ccccc}
\hline Family 1 & & & & \\
\hline$\#$ & GP & M & M & GS \\
\hline 1 & 11 & 11 & 9 & 11 \\
2 & 13 & 13 & 12 & $* 12$ \\
3 & 14 & 14 & 12 & 14 \\
4 & 8.3 & 8.3 & 7 & $* 7$ \\
5 & 31 & 31 & 36 & $* 36$ \\
6 & 20 & 20 & 15 & $* 15$ \\
7 & 11 & 11 & 9 & $* 9$ \\
8 & 8 & 8 & 9 & $* 9$ \\
9 & 13 & 13 & 9 & $* 9$ \\
10 & 14 & 14 & 11 & 14 \\
\hline
\end{tabular}

\begin{tabular}{ccccc}
\hline Family 2 & & & & \\
\hline$\#$ & GP & M & M & GS \\
\hline 1 & 10 & 10 & 12 & 10 \\
2 & 11 & 11 & 12 & 11 \\
3 & 13 & 13 & 14 & 13 \\
4 & 8.3 & 8.3 & 11 & 8.3 \\
5 & 33 & 33 & 34.1 & 33 \\
6 & 25 & 25 & 20 & $* 20$ \\
7 & 10 & 10 & 9 & $* 9$ \\
8 & 9 & 9 & 10 & $* 10$ \\
9 & 11 & 11 & 13 & $* 13$ \\
10 & 14 & 14 & 16 & $* 16$ \\
\hline
\end{tabular}

\begin{tabular}{ccccc}
\hline Family 3 & & & & \\
\hline$\#$ & GP & M & M & GS \\
\hline 1 & 11 & 11 & 11 & 11 \\
2 & 11 & 11 & 8 & 11 \\
3 & 15 & 15 & 13 & 15 \\
4 & 10 & 10 & 14 & 10 \\
5 & 33 & 33 & 34 & 33 \\
6 & 21 & 21 & 19 & 21 \\
7 & 10 & 10 & 14 & 10 \\
8 & 9 & 9 & 6 & 9 \\
9 & 9 & 9 & 11 & $* 11$ \\
10 & 14 & 14 & 15 & $* 15$ \\
\hline
\end{tabular}




\begin{tabular}{|c|c|c|c|c|c|c|}
\hline \multicolumn{7}{|l|}{ Family 4} \\
\hline$\#$ & \multicolumn{2}{|r|}{ GP } & M & \multicolumn{2}{|c|}{ M } & GS \\
\hline 1 & \multicolumn{2}{|r|}{10} & 10 & \multicolumn{2}{|c|}{10} & 10 \\
\hline 2 & \multicolumn{2}{|r|}{11} & 11 & \multicolumn{2}{|c|}{11} & 11 \\
\hline 3 & \multicolumn{2}{|r|}{14} & 14 & \multicolumn{2}{|c|}{14} & 14 \\
\hline 4 & \multicolumn{2}{|r|}{12} & 12 & \multicolumn{2}{|c|}{8.3} & 12 \\
\hline 5 & \multicolumn{2}{|r|}{31} & 31 & \multicolumn{2}{|c|}{33} & 31 \\
\hline 6 & \multicolumn{2}{|r|}{20} & 20 & \multicolumn{2}{|c|}{20} & 20 \\
\hline 7 & \multicolumn{2}{|r|}{10} & 10 & \multicolumn{2}{|c|}{9} & 10 \\
\hline 8 & \multicolumn{2}{|r|}{6} & 6 & \multicolumn{2}{|c|}{10} & 6 \\
\hline 9 & \multicolumn{2}{|r|}{12} & 12 & \multicolumn{2}{|c|}{11} & 12 \\
\hline 10 & \multicolumn{2}{|r|}{14} & 14 & \multicolumn{2}{|c|}{14} & 14 \\
\hline \multicolumn{7}{|l|}{ Family 5} \\
\hline$\#$ & GP & M & M & GS 1 & GS 2 & GS 3 \\
\hline 1 & 10 & 10 & 11 & *11 & 10 & *11 \\
\hline 2 & 11 & 11 & 12 & *12 & *12 & *12 \\
\hline 3 & 14 & 14 & 14 & 14 & 14 & 14 \\
\hline 4 & 12 & 12 & 12 & 12 & 12 & 12 \\
\hline 5 & 31 & 31 & 31 & 31 & 31 & 31 \\
\hline 6 & 20 & 20 & 20 & 20 & 20 & 20 \\
\hline 7 & 10 & 10 & 10 & 10 & 10 & 10 \\
\hline 8 & 6 & 6 & 10 & 6 & 6 & 6 \\
\hline 9 & 12 & 12 & 11 & 12 & 12 & 12 \\
\hline 10 & 14 & 14 & 16 & $* 16$ & $* 16$ & $* 16$ \\
\hline
\end{tabular}

\begin{tabular}{ccccc}
\hline Family 6 & & & & \\
\hline$\#$ & GP & M & M & GS \\
\hline 1 & 10 & 10 & 11 & $* 11$ \\
2 & 11 & 11 & 12 & $* 12$ \\
3 & 14 & 14 & 14 & 14 \\
4 & 8.3 & 8.3 & 13 & $* 13$ \\
5 & 34 & 34 & 34 & 34 \\
6 & 20 & 20 & 21 & $* 21$ \\
7 & 9 & 9 & 9 & 9 \\
8 & 8 & 8 & 11 & $* 11$ \\
9 & 9 & 9 & 11 & $* 11$ \\
10 & 15 & 15 & 15 & 15 \\
\hline
\end{tabular}




\begin{tabular}{|c|c|c|c|c|c|c|}
\hline \multicolumn{7}{|l|}{ Family 7} \\
\hline \# & GP & & M & & M & GS \\
\hline 1 & 12 & & 12 & & 12 & 12 \\
\hline 2 & 13 & & 13 & & 11 & 13 \\
\hline 3 & 14 & & 14 & & 14 & 14 \\
\hline 4 & 11 & & 11 & & 11 & 11 \\
\hline 5 & 32 & & 32 & & 34 & 32 \\
\hline 6 & 20 & & 20 & & 22 & 20 \\
\hline 7 & 9 & & 9 & & 11 & 9 \\
\hline 8 & 11 & & 11 & & 9 & 11 \\
\hline 9 & 11 & & 11 & & 12 & $* 12$ \\
\hline 10 & 15 & & 15 & & 15 & 15 \\
\hline \multicolumn{7}{|l|}{ Family 8} \\
\hline$\#$ & GP & M & & M & GS 1 & GS 2 \\
\hline 1 & 11 & 11 & & 12 & 11 & $* 12$ \\
\hline 2 & 12 & 12 & & 12 & 12 & 12 \\
\hline 3 & 17 & 17 & & 14 & $* 14$ & $* 14$ \\
\hline 4 & 11 & 11 & & 13 & $* 13$ & $* 13$ \\
\hline 5 & 33 & 33 & & 33 & 33 & 33 \\
\hline 6 & 22 & 22 & & 11 & $* 21$ & $* 21$ \\
\hline 7 & 9 & 9 & & 9 & 9 & 9 \\
\hline 8 & 12 & 12 & & 11 & $* 11$ & $* 11$ \\
\hline 9 & 13 & 13 & & 13 & 13 & 13 \\
\hline 10 & 14 & 14 & & 15 & 14 & *15 \\
\hline
\end{tabular}

\begin{tabular}{ccccccc}
\hline Family 9 & & & & & & \\
\hline$\#$ & GP & M & M & GS 1 & GS 2 & GS 3 \\
\hline 1 & 10 & 10 & 10 & 10 & 10 & 10 \\
2 & 12 & 12 & 13 & 12 & $* 13$ & 12 \\
3 & 15 & 15 & 12 & $* 12$ & $* 12$ & $* 12$ \\
4 & 13 & 13 & 14 & $* 14$ & $* 14$ & $* 14$ \\
5 & 31 & 31 & 33 & $* 33$ & $* 33$ & $* 33$ \\
6 & 21 & 21 & 20 & $* 20$ & $* 20$ & $* 20$ \\
7 & 11 & 11 & 11 & 11 & 11 & 11 \\
8 & 10 & 10 & 11 & $* 11$ & $* 11$ & $* 11$ \\
9 & 13 & 12 & 11 & $* 11$ & $* 12$ & $* 11$ \\
10 & 14 & 14 & 13 & $* 13$ & 14 & $* 13$ \\
\hline
\end{tabular}




\begin{tabular}{|c|c|c|c|c|}
\hline \multicolumn{5}{|l|}{ Family 10} \\
\hline$\#$ & GP & M & M & GS \\
\hline 1 & 11 & 11 & 10 & 11 \\
\hline 2 & 11 & 11 & 12 & 11 \\
\hline 3 & 14 & 14 & 13 & 14 \\
\hline 4 & 8.3 & 8.3 & 8.3 & 8.3 \\
\hline 5 & 33 & 33 & 33 & 33 \\
\hline 6 & 22 & 22 & 20 & 22 \\
\hline 7 & 12 & 12 & 11 & 12 \\
\hline 8 & 6 & 6 & 10 & 6 \\
\hline 9 & 12 & 12 & 13 & 12 \\
\hline 10 & 15 & 15 & 14 & 15 \\
\hline \multicolumn{5}{|l|}{ Family 11} \\
\hline$\#$ & GP & M & M & GS \\
\hline 1 & 10 & 10 & 10 & 10 \\
\hline 2 & 11 & 11 & 9 & 11 \\
\hline 3 & 15 & 15 & 12 & *12 \\
\hline 4 & 13 & 13 & 12 & $* 12$ \\
\hline 5 & 33 & 33 & 35 & *35 \\
\hline 6 & 21 & 21 & 20 & *20 \\
\hline 7 & 9 & 9 & 11 & *11 \\
\hline 8 & 6 & 6 & 9 & 6 \\
\hline 9 & 12 & 12 & 12 & 12 \\
\hline 10 & 14 & 14 & 16 & 14 \\
\hline
\end{tabular}

\begin{tabular}{ccccccccc}
\hline \multicolumn{2}{l}{ Family 12} & & & & & & & \\
\hline$\#$ & GP & GM & GM & M & M & A & A & GS \\
\hline 1 & 10 & 10 & 12 & 10 & 12 & 10 & 10 & $* 12$ \\
2 & 11 & 12 & 12 & 11 & 12 & 11 & 12 & $* 12$ \\
3 & 14 & 13 & 13 & 14 & 13 & 14 & 13 & 14 \\
4 & 14 & 8.3 & 13 & 14 & 13 & 14 & 8.3 & 14 \\
5 & 31 & 30 & 33 & 31 & 30 & 31 & 30 & 31 \\
6 & 22 & 20 & 23 & 22 & 23 & 22 & 22 & 22 \\
7 & 9 & 9 & 9 & 9 & 9 & 9 & 9 & 9 \\
8 & 8 & 10 & 10 & 8 & 10 & 8 & 10 & 8 \\
9 & 13 & 12 & 14 & 13 & 12 & 13 & 15 & $* 12$ \\
10 & 15 & 14 & 15 & 14 & 15 & 15 & 15 & 15 \\
\hline
\end{tabular}




\begin{tabular}{|c|c|c|c|c|c|c|c|c|}
\hline \multicolumn{9}{|c|}{ Family 13} \\
\hline \# & GP & GM & GM & M & M & A & A & GS \\
\hline 1 & 10 & 10 & 12 & 10 & 10 & 10 & 10 & 10 \\
\hline 2 & 11 & 12 & 12 & 11 & 12 & 11 & 12 & $* 12$ \\
\hline 3 & 14 & 13 & 13 & 14 & 13 & 13 & 14 & 14 \\
\hline 4 & 14 & 8.3 & 13 & 14 & 13 & 8.3 & 14 & 14 \\
\hline 5 & 31 & 30 & 33 & 31 & 33 & 30 & 31 & 31 \\
\hline 6 & 22 & 20 & 23 & 22 & 20 & 22 & 22 & 22 \\
\hline 7 & 11 & 12 & 12 & 11 & 12 & 9 & 9 & $* 12$ \\
\hline 8 & 8 & 10 & 10 & 8 & 10 & 8 & 10 & 8 \\
\hline 9 & 13 & 12 & 14 & 13 & 14 & 12 & 13 & 13 \\
\hline 10 & 15 & 14 & 15 & 14 & 15 & 15 & 15 & 15 \\
\hline \multicolumn{9}{|c|}{ Family 14} \\
\hline \# & & GP & & M & & M & & GS \\
\hline 1 & & 10 & & 10 & & 12 & & *12 \\
\hline 2 & & 11 & & 11 & & 12 & & *12 \\
\hline 3 & & 13 & & 13 & & 14 & & 13 \\
\hline 4 & & 13 & & 13 & & 12 & & 13 \\
\hline 5 & & 32 & & 32 & & 33 & & *33 \\
\hline 6 & & 20 & & 20 & & 22 & & 20 \\
\hline 7 & & 12 & & 12 & & 9 & & 12 \\
\hline 8 & & 8 & & 8 & & 11 & & 8 \\
\hline 9 & & 12 & & 12 & & 9 & & 12 \\
\hline 10 & & 15 & & 15 & & 15 & & 15 \\
\hline
\end{tabular}

\begin{tabular}{ccccc}
\hline Family 15 & & & & \\
\hline$\#$ & GP & M & M & GS \\
\hline 1 & 10 & 10 & 11 & 10 \\
2 & 11 & 11 & 12 & 11 \\
3 & 13 & 13 & 14 & 13 \\
4 & 13 & 13 & 12 & $* 12$ \\
5 & 32 & 32 & 33 & $* 33$ \\
6 & 20 & 20 & 22 & $* 22$ \\
7 & 12 & 12 & 9 & $* 9$ \\
8 & 8 & 8 & 11 & 8 \\
9 & 12 & 12 & 14 & 12 \\
10 & 15 & 15 & 14 & 15 \\
\hline
\end{tabular}




\begin{tabular}{|c|c|c|c|c|}
\hline \multicolumn{5}{|c|}{ Family 16} \\
\hline$\#$ & GP & M & M & GS \\
\hline 1 & 10 & 10 & 12 & *12 \\
\hline 2 & 11 & 11 & 12 & $* 12$ \\
\hline 3 & 13 & 13 & 15 & 13 \\
\hline 4 & 13 & 13 & 13 & 13 \\
\hline 5 & 32 & 32 & 36 & *36 \\
\hline 6 & 20 & 20 & 20 & 20 \\
\hline 7 & 12 & 12 & 9 & $* 9$ \\
\hline 8 & 8 & 8 & 8 & 8 \\
\hline 9 & 12 & 12 & 14 & 12 \\
\hline 10 & 15 & 15 & 14 & *14 \\
\hline \multicolumn{5}{|l|}{ Family 17} \\
\hline$\#$ & GP & M & M & GS \\
\hline 1 & 10 & 10 & 11 & 10 \\
\hline 2 & 11 & 11 & 11 & 11 \\
\hline 3 & 14 & 14 & 13 & 14 \\
\hline 4 & 12 & 12 & 11 & 12 \\
\hline 5 & 31 & 31 & 29 & 31 \\
\hline 6 & 21 & 21 & 24 & 21 \\
\hline 7 & 10 & 10 & 11 & 10 \\
\hline 8 & 8 & 8 & 10 & 8 \\
\hline 9 & 13 & 13 & 13 & 13 \\
\hline 10 & 14 & 14 & 15 & 14 \\
\hline
\end{tabular}

\begin{tabular}{ccccc}
\hline Family 18 & & & & \\
\hline$\#$ & GP & M & M & GS \\
\hline 1 & 11 & 11 & 10 & $* 10$ \\
2 & 12 & 12 & 12.1 & 12 \\
3 & 13 & 13 & 14 & $* 14$ \\
4 & 11 & 11 & 8.3 & $* 8.3$ \\
5 & 35 & 35 & 33 & $* 33$ \\
6 & 20 & 20 & 20 & 20 \\
7 & 9 & 9 & 11 & $* 11$ \\
8 & 11 & 11 & 10 & $* 10$ \\
9 & 13 & 13 & 13 & 13 \\
10 & 15 & 15 & 13 & 15 \\
\hline
\end{tabular}




\begin{tabular}{ccccccc}
\hline Family 19 & & & & & & \\
\hline$\#$ & GP & M & M & GS 1 & GS 2 & GS 3 \\
\hline 1 & 11 & 11 & 10 & 11 & 11 & 11 \\
2 & 13 & 13 & 11 & 13 & 13 & $* 11$ \\
3 & 13 & 13 & 14 & $* 14$ & 13 & $* 14$ \\
4 & 8.3 & 8.3 & 11 & $* 11$ & 8.3 & 8.3 \\
5 & 33 & 33 & 34 & $* 34$ & $* 34$ & $* 34$ \\
6 & 19 & 19 & 20 & $* 20$ & $* 20$ & 19 \\
7 & 9 & 9 & 11 & $* 11$ & $* 11$ & 9 \\
8 & 11 & 11 & 6 & $* 6$ & $* 6$ & 11 \\
9 & 15 & 15 & 9 & 15 & 15 & 15 \\
10 & 16 & 16 & 15 & 16 & 16 & 16 \\
\hline
\end{tabular}

Note: The symbols GP, M and GS denote the grandfather's, mother's and grandson's alleles, respectively. The symbol * means that there is crossing over between the two mother's alleles. In the Families 12 and 13, the GP's alleles are reconstituted from the GM's, M's and A's alleles. The symbols GM and A denote grandmother's and aunt's alleles, respectively. 


\section{Appendix B}

In this appendix we present the Tables 5-23 with the detailed linkage analysis of each family.

Table 5. Linkage analysis of family 1.

\begin{tabular}{|c|c|c|c|}
\hline Combination of Markers & LOD & $\theta_{\max }$ & $Z_{\max }^{(1)}$ \\
\hline $1-2$ & $Z=\log _{10}\left[2^{1} 2^{0} \theta^{1}(1-\theta)^{0}\right]$ & 0.5 & 0.0 \\
\hline $1-3$ & $Z=\log _{10}\left[2^{0} 2^{1} \theta^{0}(1-\theta)^{1}\right]$ & 0.0 & 0.30 \\
\hline $1-4$ & $Z=\log _{10}\left[2^{1} 2^{0} \theta^{1}(1-\theta)^{0}\right]$ & 0.5 & 0.0 \\
\hline $1-5$ & $Z=\log _{10}\left[2^{1} 2^{0} \theta^{1}(1-\theta)^{0}\right]$ & 0.5 & 0.0 \\
\hline $1-6$ & $Z=\log _{10}\left[2^{1} 2^{0} \theta^{1}(1-\theta)^{0}\right]$ & 0.5 & 0.0 \\
\hline $1-7$ & $Z=\log _{10}\left[2^{1} 2^{0} \theta^{1}(1-\theta)^{0}\right]$ & 0.5 & 0.0 \\
\hline $1-8$ & $Z=\log _{10}\left[2^{1} 2^{0} \theta^{1}(1-\theta)^{0}\right]$ & 0.5 & 0.0 \\
\hline $1-9$ & $Z=\log _{10}\left[2^{1} 2^{0} \theta^{1}(1-\theta)^{0}\right]$ & 0.5 & 0.0 \\
\hline $1-10$ & $Z=\log _{10}\left[2^{0} 2^{1} \theta^{0}(1-\theta)^{1}\right]$ & 0.0 & 0.30 \\
\hline $2-3$ & $Z=\log _{10}\left[2^{1} 2^{0} \theta^{1}(1-\theta)^{0}\right]$ & 0.5 & 0.0 \\
\hline $2-4$ & $Z=\log _{10}\left[2^{1} 2^{0} \theta^{1}(1-\theta)^{0}\right]$ & 0.5 & 0.0 \\
\hline $2-5$ & $Z=\log _{10}\left[2^{1} 2^{0} \theta^{1}(1-\theta)^{0}\right]$ & 0.5 & 0.0 \\
\hline $2-6$ & $Z=\log _{10}\left[2^{1} 2^{0} \theta^{1}(1-\theta)^{0}\right]$ & 0.5 & 0.0 \\
\hline $2-7$ & $Z=\log _{10}\left[2^{1} 2^{0} \theta^{1}(1-\theta)^{0}\right]$ & 0.5 & 0.0 \\
\hline $2-8$ & $Z=\log _{10}\left[2^{1} 2^{0} \theta^{1}(1-\theta)^{0}\right]$ & 0.5 & 0.0 \\
\hline $2-9$ & $Z=\log _{10}\left[2^{1} 2^{0} \theta^{1}(1-\theta)^{0}\right]$ & 0.5 & 0.0 \\
\hline $2-10$ & $Z=\log _{10}\left[2^{1} 2^{0} \theta^{1}(1-\theta)^{0}\right]$ & 0.5 & 0.0 \\
\hline $3-4$ & $Z=\log _{10}\left[2^{1} 2^{0} \theta^{1}(1-\theta)^{0}\right]$ & 0.5 & 0.0 \\
\hline $3-5$ & $Z=\log _{10}\left[2^{1} 2^{0} \theta^{1}(1-\theta)^{0}\right]$ & 0.5 & 0.0 \\
\hline $3-6$ & $Z=\log _{10}\left[2^{1} 2^{0} \theta^{1}(1-\theta)^{0}\right]$ & 0.5 & 0.0 \\
\hline $3-7$ & $Z=\log _{10}\left[2^{1} 2^{0} \theta^{1}(1-\theta)^{0}\right]$ & 0.5 & 0.0 \\
\hline $3-8$ & $Z=\log _{10}\left[2^{1} 2^{0} \theta^{1}(1-\theta)^{0}\right]$ & 0.5 & 0.0 \\
\hline $3-9$ & $Z=\log _{10}\left[2^{1} 2^{0} \theta^{1}(1-\theta)^{0}\right]$ & 0.5 & 0.0 \\
\hline $3-10$ & $Z=\log _{10}\left[2^{0} 2^{1} \theta^{0}(1-\theta)^{1}\right]$ & 0.0 & 0.30 \\
\hline $4-5$ & $Z=\log _{10}\left[2^{1} 2^{0} \theta^{1}(1-\theta)^{0}\right]$ & 0.5 & 0.0 \\
\hline $4-6$ & $Z=\log _{10}\left[2^{1} 2^{0} \theta^{1}(1-\theta)^{0}\right]$ & 0.5 & 0.0 \\
\hline $4-7$ & $Z=\log _{10}\left[2^{1} 2^{0} \theta^{1}(1-\theta)^{0}\right]$ & 0.5 & 0.0 \\
\hline $4-8$ & $Z=\log _{10}\left[2^{1} 2^{0} \theta^{1}(1-\theta)^{0}\right]$ & 0.5 & 0.0 \\
\hline $4-9$ & $Z=\log _{10}\left[2^{1} 2^{0} \theta^{1}(1-\theta)^{0}\right]$ & 0.5 & 0.0 \\
\hline $4-10$ & $Z=\log _{10}\left[2^{1} 2^{0} \theta^{1}(1-\theta)^{0}\right]$ & 0.5 & 0.0 \\
\hline
\end{tabular}




\section{Continued}

\begin{tabular}{|c|c|c|c|}
\hline $5-6$ & $Z=\log _{10}\left[2^{1} 2^{0} \theta^{1}(1-\theta)^{0}\right]$ & 0.5 & 0.0 \\
\hline $5-7$ & $Z=\log _{10}\left[2^{1} 2^{0} \theta^{1}(1-\theta)^{0}\right]$ & 0.5 & 0.0 \\
\hline $5-8$ & $Z=\log _{10}\left[2^{1} 2^{0} \theta^{1}(1-\theta)^{0}\right]$ & 0.5 & 0.0 \\
\hline $5-9$ & $Z=\log _{10}\left[2^{1} 2^{0} \theta^{1}(1-\theta)^{0}\right]$ & 0.5 & 0.0 \\
\hline $5-10$ & $Z=\log _{10}\left[2^{1} 2^{0} \theta^{1}(1-\theta)^{0}\right]$ & 0.5 & 0.0 \\
\hline $6-7$ & $Z=\log _{10}\left[2^{1} 2^{0} \theta^{1}(1-\theta)^{0}\right]$ & 0.5 & 0.0 \\
\hline $6-8$ & $Z=\log _{10}\left[2^{1} 2^{0} \theta^{1}(1-\theta)^{0}\right]$ & 0.5 & 0.0 \\
\hline $6-9$ & $Z=\log _{10}\left[2^{1} 2^{0} \theta^{1}(1-\theta)^{0}\right]$ & 0.5 & 0.0 \\
\hline $6-10$ & $Z=\log _{10}\left[2^{1} 2^{0} \theta^{1}(1-\theta)^{0}\right]$ & 0.5 & 0.0 \\
\hline $7-8$ & $Z=\log _{10}\left[2^{1} 2^{0} \theta^{1}(1-\theta)^{0}\right]$ & 0.5 & 0.0 \\
\hline $7-9$ & $Z=\log _{10}\left[2^{1} 2^{0} \theta^{1}(1-\theta)^{0}\right]$ & 0.5 & 0.0 \\
\hline $7-10$ & $Z=\log _{10}\left[2^{1} 2^{0} \theta^{1}(1-\theta)^{0}\right]$ & 0.5 & 0.0 \\
\hline $8-9$ & $Z=\log _{10}\left[2^{1} 2^{0} \theta^{1}(1-\theta)^{0}\right]$ & 0.5 & 0.0 \\
\hline $8-10$ & $Z=\log _{10}\left[2^{1} 2^{0} \theta^{1}(1-\theta)^{0}\right]$ & 0.5 & 0.0 \\
\hline
\end{tabular}

Table 6. Linkage analysis of family 2.

\begin{tabular}{|c|c|c|c|}
\hline $\begin{array}{l}\text { Combination } \\
\text { of Markers }\end{array}$ & LOD & $\theta_{\max }$ & $Z_{\max }^{(2)}$ \\
\hline $1-2$ & $Z=\log _{10}\left[2^{0} 2^{1} \theta^{0}(1-\theta)^{1}\right]$ & 0.0 & 0.30 \\
\hline $1-3$ & $Z=\log _{10}\left[2^{0} 2^{1} \theta^{0}(1-\theta)^{1}\right]$ & 0.0 & 0.30 \\
\hline $1-4$ & $Z=\log _{10}\left[2^{0} 2^{1} \theta^{0}(1-\theta)^{1}\right]$ & 0.0 & 0.30 \\
\hline $1-5$ & $Z=\log _{10}\left[2^{0} 2^{1} \theta^{0}(1-\theta)^{1}\right]$ & 0.0 & 0.30 \\
\hline $1-6$ & $Z=\log _{10}\left[2^{1} 2^{0} \theta^{1}(1-\theta)^{0}\right]$ & 0.5 & 0.0 \\
\hline $1-7$ & $Z=\log _{10}\left[2^{1} 2^{0} \theta^{1}(1-\theta)^{0}\right]$ & 0.5 & 0.0 \\
\hline $1-8$ & $Z=\log _{10}\left[2^{1} 2^{0} \theta^{1}(1-\theta)^{0}\right]$ & 0.5 & 0.0 \\
\hline $1-9$ & $Z=\log _{10}\left[2^{1} 2^{0} \theta^{1}(1-\theta)^{0}\right]$ & 0.5 & 0.0 \\
\hline $1-10$ & $Z=\log _{10}\left[2^{1} 2^{0} \theta^{1}(1-\theta)^{0}\right]$ & 0.5 & 0.0 \\
\hline $2-3$ & $Z=\log _{10}\left[2^{0} 2^{1} \theta^{0}(1-\theta)^{1}\right]$ & 0.0 & 0.30 \\
\hline $2-4$ & $Z=\log _{10}\left[2^{0} 2^{1} \theta^{0}(1-\theta)^{1}\right]$ & 0.0 & 0.30 \\
\hline $2-5$ & $Z=\log _{10}\left[2^{0} 2^{1} \theta^{0}(1-\theta)^{1}\right]$ & 0.0 & 0.30 \\
\hline $2-6$ & $Z=\log _{10}\left[2^{1} 2^{0} \theta^{1}(1-\theta)^{0}\right]$ & 0.5 & 0.0 \\
\hline $2-7$ & $Z=\log _{10}\left[2^{1} 2^{0} \theta^{1}(1-\theta)^{0}\right]$ & 0.5 & 0.0 \\
\hline $2-8$ & $Z=\log _{10}\left[2^{1} 2^{0} \theta^{1}(1-\theta)^{0}\right]$ & 0.5 & 0.0 \\
\hline $2-9$ & $Z=\log _{10}\left[2^{1} 2^{0} \theta^{1}(1-\theta)^{0}\right]$ & 0.5 & 0.0 \\
\hline $2-10$ & $Z=\log _{10}\left[2^{1} 2^{0} \theta^{1}(1-\theta)^{0}\right]$ & 0.5 & 0.0 \\
\hline
\end{tabular}




\section{Continued}

\begin{tabular}{|c|c|c|c|}
\hline $3-4$ & $Z=\log _{10}\left[2^{0} 2^{1} \theta^{0}(1-\theta)^{1}\right]$ & 0.0 & 0.30 \\
\hline $3-5$ & $Z=\log _{10}\left[2^{0} 2^{1} \theta^{0}(1-\theta)^{1}\right]$ & 0.0 & 0.30 \\
\hline $3-6$ & $Z=\log _{10}\left[2^{1} 2^{0} \theta^{1}(1-\theta)^{0}\right]$ & 0.5 & 0.0 \\
\hline $3-7$ & $Z=\log _{10}\left[2^{1} 2^{0} \theta^{1}(1-\theta)^{0}\right]$ & 0.5 & 0.0 \\
\hline $3-8$ & $Z=\log _{10}\left[2^{1} 2^{0} \theta^{1}(1-\theta)^{0}\right]$ & 0.5 & 0.0 \\
\hline $3-9$ & $Z=\log _{10}\left[2^{1} 2^{0} \theta^{1}(1-\theta)^{0}\right]$ & 0.5 & 0.0 \\
\hline $3-10$ & $Z=\log _{10}\left[2^{1} 2^{0} \theta^{1}(1-\theta)^{0}\right]$ & 0.5 & 0.0 \\
\hline $4-5$ & $Z=\log _{10}\left[2^{0} 2^{1} \theta^{0}(1-\theta)^{1}\right]$ & 0.0 & 0.30 \\
\hline $4-6$ & $Z=\log _{10}\left[2^{1} 2^{0} \theta^{1}(1-\theta)^{0}\right]$ & 0.5 & 0.0 \\
\hline $4-7$ & $Z=\log _{10}\left[2^{1} 2^{0} \theta^{1}(1-\theta)^{0}\right]$ & 0.5 & 0.0 \\
\hline $4-8$ & $Z=\log _{10}\left[2^{1} 2^{0} \theta^{1}(1-\theta)^{0}\right]$ & 0.5 & 0.0 \\
\hline $4-9$ & $Z=\log _{10}\left[2^{1} 2^{0} \theta^{1}(1-\theta)^{0}\right]$ & 0.5 & 0.0 \\
\hline $4-10$ & $Z=\log _{10}\left[2^{1} 2^{0} \theta^{1}(1-\theta)^{0}\right]$ & 0.5 & 0.0 \\
\hline $5-6$ & $Z=\log _{10}\left[2^{1} 2^{0} \theta^{1}(1-\theta)^{0}\right]$ & 0.5 & 0.0 \\
\hline $5-7$ & $Z=\log _{10}\left[2^{1} 2^{0} \theta^{1}(1-\theta)^{0}\right]$ & 0.5 & 0.0 \\
\hline $5-8$ & $Z=\log _{10}\left[2^{1} 2^{0} \theta^{1}(1-\theta)^{0}\right]$ & 0.5 & 0.0 \\
\hline $5-9$ & $Z=\log _{10}\left[2^{1} 2^{0} \theta^{1}(1-\theta)^{0}\right]$ & 0.5 & 0.0 \\
\hline $5-10$ & $Z=\log _{10}\left[2^{1} 2^{0} \theta^{1}(1-\theta)^{0}\right]$ & 0.5 & 0.0 \\
\hline $6-7$ & $Z=\log _{10}\left[2^{1} 2^{0} \theta^{1}(1-\theta)^{0}\right]$ & 0.5 & 0.0 \\
\hline $6-8$ & $Z=\log _{10}\left[2^{1} 2^{0} \theta^{1}(1-\theta)^{0}\right]$ & 0.5 & 0.0 \\
\hline $6-9$ & $Z=\log _{10}\left[2^{1} 2^{0} \theta^{1}(1-\theta)^{0}\right]$ & 0.5 & 0.0 \\
\hline $6-10$ & $Z=\log _{10}\left[2^{1} 2^{0} \theta^{1}(1-\theta)^{0}\right]$ & 0.5 & 0.0 \\
\hline $7-8$ & $Z=\log _{10}\left[2^{1} 2^{0} \theta^{1}(1-\theta)^{0}\right]$ & 0.5 & 0.0 \\
\hline $7-9$ & $Z=\log _{10}\left[2^{1} 2^{0} \theta^{1}(1-\theta)^{0}\right]$ & 0.5 & 0.0 \\
\hline $7-10$ & $Z=\log _{10}\left[2^{1} 2^{0} \theta^{1}(1-\theta)^{0}\right]$ & 0.5 & 0.0 \\
\hline $8-9$ & $Z=\log _{10}\left[2^{1} 2^{0} \theta^{1}(1-\theta)^{0}\right]$ & 0.5 & 0.0 \\
\hline $8-10$ & $Z=\log _{10}\left[2^{1} 2^{0} \theta^{1}(1-\theta)^{0}\right]$ & 0.5 & 0.0 \\
\hline
\end{tabular}

Table 7. Linkage analysis of family 3.

\begin{tabular}{cccc}
\hline Combination of Markers & LOD & $\theta_{\text {max }}$ & $Z_{\text {max }}^{(3)}$ \\
\hline $1-2$ & $Z=\log _{10}\left[2^{0} 2^{1} \theta^{0}(1-\theta)^{1}\right]$ & 0.0 & 0.30 \\
$1-3$ & $Z=\log _{10}\left[2^{0} 2^{1} \theta^{0}(1-\theta)^{1}\right]$ & 0.0 & 0.30 \\
$1-4$ & $Z=\log _{10}\left[2^{0} 2^{1} \theta^{0}(1-\theta)^{1}\right]$ & 0.0 & 0.30 \\
$1-5$ & $Z=\log _{10}\left[2^{0} 2^{1} \theta^{0}(1-\theta)^{1}\right]$ & 0.0 & 0.30 \\
$1-6$ & $Z=\log _{10}\left[2^{0} 2^{1} \theta^{0}(1-\theta)^{1}\right]$ & 0.0 & 0.30 \\
\hline
\end{tabular}




\section{Continued}

\begin{tabular}{|c|c|c|c|}
\hline $1-7$ & $Z=\log _{10}\left[2^{0} 2^{1} \theta^{0}(1-\theta)^{1}\right]$ & 0.0 & 0.30 \\
\hline $1-8$ & $Z=\log _{10}\left[2^{0} 2^{1} \theta^{0}(1-\theta)^{1}\right]$ & 0.0 & 0.30 \\
\hline $1-9$ & $Z=\log _{10}\left[2^{1} 2^{0} \theta^{1}(1-\theta)^{0}\right]$ & 0.5 & 0.0 \\
\hline $1-10$ & $Z=\log _{10}\left[2^{1} 2^{0} \theta^{1}(1-\theta)^{0}\right]$ & 0.5 & 0.0 \\
\hline $2-3$ & $Z=\log _{10}\left[2^{0} 2^{1} \theta^{0}(1-\theta)^{1}\right]$ & 0.0 & 0.30 \\
\hline $2-4$ & $Z=\log _{10}\left[2^{0} 2^{1} \theta^{0}(1-\theta)^{1}\right]$ & 0.0 & 0.30 \\
\hline $2-5$ & $Z=\log _{10}\left[2^{0} 2^{1} \theta^{0}(1-\theta)^{1}\right]$ & 0.0 & 0.30 \\
\hline $2-6$ & $Z=\log _{10}\left[2^{0} 2^{1} \theta^{0}(1-\theta)^{1}\right]$ & 0.0 & 0.30 \\
\hline $2-7$ & $Z=\log _{10}\left[2^{0} 2^{1} \theta^{0}(1-\theta)^{1}\right]$ & 0.0 & 0.30 \\
\hline $2-8$ & $Z=\log _{10}\left[2^{0} 2^{1} \theta^{0}(1-\theta)^{1}\right]$ & 0.0 & 0.30 \\
\hline $2-9$ & $Z=\log _{10}\left[2^{1} 2^{0} \theta^{1}(1-\theta)^{0}\right]$ & 0.5 & 0.0 \\
\hline $2-10$ & $Z=\log _{10}\left[2^{1} 2^{0} \theta^{1}(1-\theta)^{0}\right]$ & 0.5 & 0.0 \\
\hline $3-4$ & $Z=\log _{10}\left[2^{0} 2^{1} \theta^{0}(1-\theta)^{1}\right]$ & 0.0 & 0.30 \\
\hline $3-5$ & $Z=\log _{10}\left[2^{0} 2^{1} \theta^{0}(1-\theta)^{1}\right]$ & 0.0 & 0.30 \\
\hline $3-6$ & $Z=\log _{10}\left[2^{0} 2^{1} \theta^{0}(1-\theta)^{1}\right]$ & 0.0 & 0.30 \\
\hline $3-7$ & $Z=\log _{10}\left[2^{0} 2^{1} \theta^{0}(1-\theta)^{1}\right]$ & 0.0 & 0.30 \\
\hline $3-8$ & $Z=\log _{10}\left[2^{0} 2^{1} \theta^{0}(1-\theta)^{1}\right]$ & 0.0 & 0.30 \\
\hline $3-9$ & $Z=\log _{10}\left[2^{1} 2^{0} \theta^{1}(1-\theta)^{0}\right]$ & 0.5 & 0.0 \\
\hline $3-10$ & $Z=\log _{10}\left[2^{1} 2^{0} \theta^{1}(1-\theta)^{0}\right]$ & 0.5 & 0.0 \\
\hline $4-5$ & $Z=\log _{10}\left[2^{0} 2^{1} \theta^{0}(1-\theta)^{1}\right]$ & 0.0 & 0.30 \\
\hline $4-6$ & $Z=\log _{10}\left[2^{0} 2^{1} \theta^{0}(1-\theta)^{1}\right]$ & 0.0 & 0.30 \\
\hline $4-7$ & $Z=\log _{10}\left[2^{0} 2^{1} \theta^{0}(1-\theta)^{1}\right]$ & 0.0 & 0.30 \\
\hline $4-8$ & $Z=\log _{10}\left[2^{0} 2^{1} \theta^{0}(1-\theta)^{1}\right]$ & 0.0 & 0.30 \\
\hline $4-9$ & $Z=\log _{10}\left[2^{1} 2^{0} \theta^{1}(1-\theta)^{0}\right]$ & 0.5 & 0.0 \\
\hline $4-10$ & $Z=\log _{10}\left[2^{1} 2^{0} \theta^{1}(1-\theta)^{0}\right]$ & 0.5 & 0.0 \\
\hline $5-6$ & $Z=\log _{10}\left[2^{0} 2^{1} \theta^{0}(1-\theta)^{1}\right]$ & 0.0 & 0.30 \\
\hline $5-7$ & $Z=\log _{10}\left[2^{0} 2^{1} \theta^{0}(1-\theta)^{1}\right]$ & 0.0 & 0.30 \\
\hline $5-8$ & $Z=\log _{10}\left[2^{0} 2^{1} \theta^{0}(1-\theta)^{1}\right]$ & 0.0 & 0.30 \\
\hline $5-9$ & $Z=\log _{10}\left[2^{1} 2^{0} \theta^{1}(1-\theta)^{0}\right]$ & 0.5 & 0.0 \\
\hline $5-10$ & $Z=\log _{10}\left[2^{1} 2^{0} \theta^{1}(1-\theta)^{0}\right]$ & 0.5 & 0.0 \\
\hline $6-7$ & $Z=\log _{10}\left[2^{0} 2^{1} \theta^{0}(1-\theta)^{1}\right]$ & 0.0 & 0.30 \\
\hline $6-8$ & $Z=\log _{10}\left[2^{0} 2^{1} \theta^{0}(1-\theta)^{1}\right]$ & 0.0 & 0.30 \\
\hline $6-9$ & $Z=\log _{10}\left[2^{1} 2^{0} \theta^{1}(1-\theta)^{0}\right]$ & 0.5 & 0.0 \\
\hline $6-10$ & $Z=\log _{10}\left[2^{1} 2^{0} \theta^{1}(1-\theta)^{0}\right]$ & 0.5 & 0.0 \\
\hline
\end{tabular}




\section{Continued}

$\begin{array}{llll}7-8 & Z=\log _{10}\left[2^{0} 2^{1} \theta^{0}(1-\theta)^{1}\right] & 0.0 & 0.30 \\ 7-9 & Z=\log _{10}\left[2^{1} 2^{0} \theta^{1}(1-\theta)^{0}\right] & 0.5 & 0.0 \\ 7-10 & Z=\log _{10}\left[2^{1} 2^{0} \theta^{1}(1-\theta)^{0}\right] & 0.5 & 0.0 \\ 8-9 & Z=\log _{10}\left[2^{1} 2^{0} \theta^{1}(1-\theta)^{0}\right] & 0.5 & 0.0 \\ 8-10 & Z=\log _{10}\left[2^{1} 2^{0} \theta^{1}(1-\theta)^{0}\right] & 0.5 & 0.0\end{array}$

Table 8. Linkage analysis of family 4.

\begin{tabular}{|c|c|c|c|}
\hline Combination of Markers & LOD & $\theta_{\max }$ & $Z_{\max }^{(4)}$ \\
\hline $1-2$ & $Z=\log _{10}\left[2^{0} 2^{1} \theta^{0}(1-\theta)^{1}\right]$ & 0.0 & 0.30 \\
\hline $1-3$ & $Z=\log _{10}\left[2^{0} 2^{1} \theta^{0}(1-\theta)^{1}\right]$ & 0.0 & 0.30 \\
\hline $1-4$ & $Z=\log _{10}\left[2^{0} 2^{1} \theta^{0}(1-\theta)^{1}\right]$ & 0.0 & 0.30 \\
\hline $1-5$ & $Z=\log _{10}\left[2^{0} 2^{1} \theta^{0}(1-\theta)^{1}\right]$ & 0.0 & 0.30 \\
\hline $1-6$ & $Z=\log _{10}\left[2^{0} 2^{1} \theta^{0}(1-\theta)^{1}\right]$ & 0.0 & 0.30 \\
\hline $1-7$ & $Z=\log _{10}\left[2^{0} 2^{1} \theta^{0}(1-\theta)^{1}\right]$ & 0.0 & 0.30 \\
\hline $1-8$ & $Z=\log _{10}\left[2^{0} 2^{1} \theta^{0}(1-\theta)^{1}\right]$ & 0.0 & 0.30 \\
\hline $1-9$ & $Z=\log _{10}\left[2^{0} 2^{1} \theta^{0}(1-\theta)^{1}\right]$ & 0.0 & 0.30 \\
\hline $1-10$ & $Z=\log _{10}\left[2^{0} 2^{1} \theta^{0}(1-\theta)^{1}\right]$ & 0.0 & 0.30 \\
\hline $2-3$ & $Z=\log _{10}\left[2^{0} 2^{1} \theta^{0}(1-\theta)^{1}\right]$ & 0.0 & 0.30 \\
\hline $2-4$ & $Z=\log _{10}\left[2^{0} 2^{1} \theta^{0}(1-\theta)^{1}\right]$ & 0.0 & 0.30 \\
\hline $2-5$ & $Z=\log _{10}\left[2^{0} 2^{1} \theta^{0}(1-\theta)^{1}\right]$ & 0.0 & 0.30 \\
\hline $2-6$ & $Z=\log _{10}\left[2^{0} 2^{1} \theta^{0}(1-\theta)^{1}\right]$ & 0.0 & 0.30 \\
\hline $2-7$ & $Z=\log _{10}\left[2^{0} 2^{1} \theta^{0}(1-\theta)^{1}\right]$ & 0.0 & 0.30 \\
\hline $2-8$ & $Z=\log _{10}\left[2^{0} 2^{1} \theta^{0}(1-\theta)^{1}\right]$ & 0.0 & 0.30 \\
\hline $2-9$ & $Z=\log _{10}\left[2^{0} 2^{1} \theta^{0}(1-\theta)^{1}\right]$ & 0.0 & 0.30 \\
\hline $2-10$ & $Z=\log _{10}\left[2^{0} 2^{1} \theta^{0}(1-\theta)^{1}\right]$ & 0.0 & 0.30 \\
\hline $3-4$ & $Z=\log _{10}\left[2^{0} 2^{1} \theta^{0}(1-\theta)^{1}\right]$ & 0.0 & 0.30 \\
\hline $3-5$ & $Z=\log _{10}\left[2^{0} 2^{1} \theta^{0}(1-\theta)^{1}\right]$ & 0.0 & 0.30 \\
\hline $3-6$ & $Z=\log _{10}\left[2^{0} 2^{1} \theta^{0}(1-\theta)^{1}\right]$ & 0.0 & 0.30 \\
\hline $3-7$ & $Z=\log _{10}\left[2^{0} 2^{1} \theta^{0}(1-\theta)^{1}\right]$ & 0.0 & 0.30 \\
\hline $3-8$ & $Z=\log _{10}\left[2^{0} 2^{1} \theta^{0}(1-\theta)^{1}\right]$ & 0.0 & 0.30 \\
\hline $3-9$ & $Z=\log _{10}\left[2^{0} 2^{1} \theta^{0}(1-\theta)^{1}\right]$ & 0.0 & 0.30 \\
\hline $3-10$ & $Z=\log _{10}\left[2^{0} 2^{1} \theta^{0}(1-\theta)^{1}\right]$ & 0.0 & 0.30 \\
\hline $4-5$ & $Z=\log _{10}\left[2^{0} 2^{1} \theta^{0}(1-\theta)^{1}\right]$ & 0.0 & 0.30 \\
\hline $4-6$ & $Z=\log _{10}\left[2^{0} 2^{1} \theta^{0}(1-\theta)^{1}\right]$ & 0.0 & 0.30 \\
\hline
\end{tabular}




\section{Continued}

$\begin{array}{llll}4-7 & Z=\log _{10}\left[2^{0} 2^{1} \theta^{0}(1-\theta)^{1}\right] & 0.0 & 0.30 \\ 4-8 & Z=\log _{10}\left[2^{0} 2^{1} \theta^{0}(1-\theta)^{1}\right] & 0.0 & 0.30 \\ 4-9 & Z=\log _{10}\left[2^{0} 2^{1} \theta^{0}(1-\theta)^{1}\right] & 0.0 & 0.30 \\ 4-10 & Z=\log _{10}\left[2^{0} 2^{1} \theta^{0}(1-\theta)^{1}\right] & 0.0 & 0.30 \\ 5-6 & Z=\log _{10}\left[2^{0} 2^{1} \theta^{0}(1-\theta)^{1}\right] & 0.0 & 0.30 \\ 5-7 & Z=\log _{10}\left[2^{0} 2^{1} \theta^{0}(1-\theta)^{1}\right] & 0.0 & 0.30 \\ 5-8 & Z=\log _{10}\left[2^{0} 2^{1} \theta^{0}(1-\theta)^{1}\right] & 0.0 & 0.30 \\ 5-9 & Z=\log _{10}\left[2^{0} 2^{1} \theta^{0}(1-\theta)^{1}\right] & 0.0 & 0.30 \\ 5-10 & Z=\log _{10}\left[2^{0} 2^{1} \theta^{0}(1-\theta)^{1}\right] & 0.0 & 0.30 \\ 6-7 & Z=\log _{10}\left[2^{0} 2^{1} \theta^{0}(1-\theta)^{1}\right] & 0.0 & 0.30 \\ 6-8 & Z=\log _{10}\left[2^{0} 2^{1} \theta^{0}(1-\theta)^{1}\right] & 0.0 & 0.30 \\ 6-9 & Z=\log _{10}\left[2^{0} 2^{1} \theta^{0}(1-\theta)^{1}\right] & 0.0 & 0.30 \\ 6-10 & Z=\log _{10}\left[2^{0} 2^{1} \theta^{0}(1-\theta)^{1}\right] & 0.0 & 0.30 \\ 7-8 & Z=\log _{10}\left[2^{0} 2^{1} \theta^{0}(1-\theta)^{1}\right] & 0.0 & 0.30 \\ 7-9 & Z=\log _{10}\left[2^{0} 2^{1} \theta^{0}(1-\theta)^{1}\right] & 0.0 & 0.30\end{array}$

Table 9. Linkage analysis of family 5.

\begin{tabular}{cccc}
\hline Combination of Markers & LOD & $\theta_{\text {max }}$ & $Z_{\text {max }}^{(5)}$ \\
\hline $1-2$ & $Z=\log _{10}\left[2^{3} 2^{0} \theta^{3}(1-\theta)^{0}\right]$ & 0.5 & 0.0 \\
$1-3$ & $Z=\log _{10}\left[2^{2} 2^{1} \theta^{2}(1-\theta)^{1}\right]$ & 0.5 & 0.0 \\
$1-4$ & $Z=\log _{10}\left[2^{2} 2^{1} \theta^{2}(1-\theta)^{1}\right]$ & 0.5 & 0.0 \\
$1-5$ & $Z=\log _{10}\left[2^{2} 2^{1} \theta^{2}(1-\theta)^{1}\right]$ & 0.5 & 0.0 \\
$1-6$ & $Z=\log _{10}\left[2^{2} 2^{1} \theta^{2}(1-\theta)^{1}\right]$ & 0.5 & 0.0 \\
$1-7$ & $Z=\log _{10}\left[2^{2} 2^{1} \theta^{2}(1-\theta)^{1}\right]$ & 0.5 & 0.0 \\
$1-8$ & $Z=\log _{10}\left[2^{2} 2^{1} \theta^{2}(1-\theta)^{1}\right]$ & 0.5 & 0.0 \\
$1-9$ & $Z=\log _{10}\left[2^{2} 2^{1} \theta^{2}(1-\theta)^{1}\right]$ \\
$1-10$ & $Z=\log _{10}\left[2^{3} 2^{0} \theta^{3}(1-\theta)^{0}\right]$ & 0.5 & 0.0 \\
$2-3$ & $Z=\log _{10}\left[2^{3} 2^{0} \theta^{3}(1-\theta)^{0}\right]$ \\
$Z-4$ & $Z=\log _{10}\left[2^{3} 2^{0} \theta^{3}(1-\theta)^{0}\right]$ \\
$Z=\log _{10}\left[2^{3} 2^{0} \theta^{3}(1-\theta)^{0}\right]$ & 0.5 & 0.0 \\
$2-5$ & $Z=\log _{10}\left[2^{3} 2^{0} \theta^{3}(1-\theta)^{0}\right]$ & 0.5 & 0.0 \\
\hline
\end{tabular}




\section{Continued}

\begin{tabular}{|c|c|c|c|}
\hline $2-7$ & $Z=\log _{10}\left[2^{3} 2^{0} \theta^{3}(1-\theta)^{0}\right]$ & 0.5 & 0.0 \\
\hline $2-8$ & $Z=\log _{10}\left[2^{3} 2^{0} \theta^{3}(1-\theta)^{0}\right]$ & 0.5 & 0.0 \\
\hline $2-9$ & $Z=\log _{10}\left[2^{3} 2^{0} \theta^{3}(1-\theta)^{0}\right]$ & 0.5 & 0.0 \\
\hline $2-10$ & $Z=\log _{10}\left[2^{3} 2^{0} \theta^{3}(1-\theta)^{0}\right]$ & 0.5 & 0.0 \\
\hline $3-4$ & $Z=\log _{10}\left[2^{0} 2^{3} \theta^{0}(1-\theta)^{3}\right]$ & 0.5 & 0.0 \\
\hline $3-5$ & $Z=\log _{10}\left[2^{0} 2^{3} \theta^{0}(1-\theta)^{3}\right]$ & 0.5 & 0.0 \\
\hline $3-6$ & $Z=\log _{10}\left[2^{0} 2^{3} \theta^{0}(1-\theta)^{3}\right]$ & 0.5 & 0.0 \\
\hline $3-7$ & $Z=\log _{10}\left[2^{0} 2^{3} \theta^{0}(1-\theta)^{3}\right]$ & 0.5 & 0.0 \\
\hline $3-8$ & $Z=\log _{10}\left[2^{0} 2^{3} \theta^{0}(1-\theta)^{3}\right]$ & 0.5 & 0.0 \\
\hline $3-9$ & $Z=\log _{10}\left[2^{0} 2^{3} \theta^{0}(1-\theta)^{3}\right]$ & 0.5 & 0.0 \\
\hline $3-10$ & $Z=\log _{10}\left[2^{3} 2^{0} \theta^{3}(1-\theta)^{0}\right]$ & 0.5 & 0.0 \\
\hline $4-5$ & $Z=\log _{10}\left[2^{0} 2^{3} \theta^{0}(1-\theta)^{3}\right]$ & 0.5 & 0.0 \\
\hline $4-6$ & $Z=\log _{10}\left[2^{0} 2^{3} \theta^{0}(1-\theta)^{3}\right]$ & 0.5 & 0.0 \\
\hline $4-7$ & $Z=\log _{10}\left[2^{0} 2^{3} \theta^{0}(1-\theta)^{3}\right]$ & 0.5 & 0.0 \\
\hline $4-8$ & $Z=\log _{10}\left[2^{0} 2^{3} \theta^{0}(1-\theta)^{3}\right]$ & 0.5 & 0.0 \\
\hline $4-9$ & $Z=\log _{10}\left[2^{0} 2^{3} \theta^{0}(1-\theta)^{3}\right]$ & 0.5 & 0.0 \\
\hline $4-10$ & $Z=\log _{10}\left[2^{3} 2^{0} \theta^{3}(1-\theta)^{0}\right]$ & 0.5 & 0.0 \\
\hline $5-6$ & $Z=\log _{10}\left[2^{0} 2^{3} \theta^{0}(1-\theta)^{3}\right]$ & 0.5 & 0.0 \\
\hline $5-7$ & $Z=\log _{10}\left[2^{0} 2^{3} \theta^{0}(1-\theta)^{3}\right]$ & 0.5 & 0.0 \\
\hline $5-8$ & $Z=\log _{10}\left[2^{0} 2^{3} \theta^{0}(1-\theta)^{3}\right]$ & 0.5 & 0.0 \\
\hline $5-9$ & $Z=\log _{10}\left[2^{0} 2^{3} \theta^{0}(1-\theta)^{3}\right]$ & 0.5 & 0.0 \\
\hline $5-10$ & $Z=\log _{10}\left[2^{3} 2^{0} \theta^{3}(1-\theta)^{0}\right]$ & 0.5 & 0.0 \\
\hline $6-7$ & $Z=\log _{10}\left[2^{0} 2^{1} \theta^{0}(1-\theta)^{1}\right]$ & 0.5 & 0.0 \\
\hline $6-8$ & $Z=\log _{10}\left[2^{0} 2^{1} \theta^{0}(1-\theta)^{1}\right]$ & 0.5 & 0.0 \\
\hline $6-9$ & $Z=\log _{10}\left[2^{0} 2^{1} \theta^{0}(1-\theta)^{1}\right]$ & 0.5 & 0.0 \\
\hline $6-10$ & $Z=\log _{10}\left[2^{1} 2^{0} \theta^{1}(1-\theta)^{0}\right]$ & 0.5 & 0.0 \\
\hline $7-8$ & $Z=\log _{10}\left[2^{0} 2^{3} \theta^{0}(1-\theta)^{3}\right]$ & 0.5 & 0.0 \\
\hline $7-9$ & $Z=\log _{10}\left[2^{0} 2^{3} \theta^{0}(1-\theta)^{3}\right]$ & 0.5 & 0.0 \\
\hline $7-10$ & $Z=\log _{10}\left[2^{3} 2^{0} \theta^{3}(1-\theta)^{0}\right]$ & 0.5 & 0.0 \\
\hline $8-9$ & $Z=\log _{10}\left[2^{0} 2^{3} \theta^{0}(1-\theta)^{3}\right]$ & 0.5 & 0.0 \\
\hline $8-10$ & $Z=\log _{10}\left[2^{3} 2^{0} \theta^{3}(1-\theta)^{0}\right]$ & 0.5 & 0.0 \\
\hline
\end{tabular}


Table 10. Linkage analysis of family 6.

\begin{tabular}{|c|c|c|c|}
\hline Combination of Markers & LOD & $\theta_{\max }$ & $Z_{\max }^{(6)}$ \\
\hline $1-2$ & $Z=\log _{10}\left[2^{1} 2^{0} \theta^{1}(1-\theta)^{0}\right]$ & 0.5 & 0.0 \\
\hline $1-3$ & $Z=\log _{10}\left[2^{1} 2^{0} \theta^{1}(1-\theta)^{0}\right]$ & 0.5 & 0.0 \\
\hline $1-4$ & $Z=\log _{10}\left[2^{1} 2^{0} \theta^{1}(1-\theta)^{0}\right]$ & 0.5 & 0.0 \\
\hline $1-5$ & $Z=\log _{10}\left[2^{1} 2^{0} \theta^{1}(1-\theta)^{0}\right]$ & 0.5 & 0.0 \\
\hline $1-6$ & $Z=\log _{10}\left[2^{1} 2^{0} \theta^{1}(1-\theta)^{0}\right]$ & 0.5 & 0.0 \\
\hline $1-7$ & $Z=\log _{10}\left[2^{1} 2^{0} \theta^{1}(1-\theta)^{0}\right]$ & 0.5 & 0.0 \\
\hline $1-8$ & $Z=\log _{10}\left[2^{1} 2^{0} \theta^{1}(1-\theta)^{0}\right]$ & 0.5 & 0.0 \\
\hline $1-9$ & $Z=\log _{10}\left[2^{1} 2^{0} \theta^{1}(1-\theta)^{0}\right]$ & 0.5 & 0.0 \\
\hline $1-10$ & $Z=\log _{10}\left[2^{1} 2^{0} \theta^{1}(1-\theta)^{0}\right]$ & 0.5 & 0.0 \\
\hline $2-3$ & $Z=\log _{10}\left[2^{1} 2^{0} \theta^{1}(1-\theta)^{0}\right]$ & 0.5 & 0.0 \\
\hline $2-4$ & $Z=\log _{10}\left[2^{1} 2^{0} \theta^{1}(1-\theta)^{0}\right]$ & 0.5 & 0.0 \\
\hline $2-5$ & $Z=\log _{10}\left[2^{1} 2^{0} \theta^{1}(1-\theta)^{0}\right]$ & 0.5 & 0.0 \\
\hline $2-6$ & $Z=\log _{10}\left[2^{1} 2^{0} \theta^{1}(1-\theta)^{0}\right]$ & 0.5 & 0.0 \\
\hline $2-7$ & $Z=\log _{10}\left[2^{1} 2^{0} \theta^{1}(1-\theta)^{0}\right]$ & 0.5 & 0.0 \\
\hline $2-8$ & $Z=\log _{10}\left[2^{1} 2^{0} \theta^{1}(1-\theta)^{0}\right]$ & 0.5 & 0.0 \\
\hline $2-9$ & $Z=\log _{10}\left[2^{1} 2^{0} \theta^{1}(1-\theta)^{0}\right]$ & 0.5 & 0.0 \\
\hline $2-10$ & $Z=\log _{10}\left[2^{1} 2^{0} \theta^{1}(1-\theta)^{0}\right]$ & 0.5 & 0.0 \\
\hline $3-4$ & $Z=\log _{10}\left[2^{1} 2^{0} \theta^{1}(1-\theta)^{0}\right]$ & 0.5 & 0.0 \\
\hline $3-5$ & $Z=\log _{10}\left[2^{0} 2^{1} \theta^{0}(1-\theta)^{1}\right]$ & 0.0 & 0.30 \\
\hline $3-6$ & $Z=\log _{10}\left[2^{1} 2^{0} \theta^{1}(1-\theta)^{0}\right]$ & 0.5 & 0.0 \\
\hline $3-7$ & $Z=\log _{10}\left[2^{0} 2^{1} \theta^{0}(1-\theta)^{1}\right]$ & 0.0 & 0.30 \\
\hline $3-8$ & $Z=\log _{10}\left[2^{1} 2^{0} \theta^{1}(1-\theta)^{0}\right]$ & 0.5 & 0.0 \\
\hline $3-9$ & $Z=\log _{10}\left[2^{0} 2^{1} \theta^{0}(1-\theta)^{1}\right]$ & 0.0 & 0.30 \\
\hline $3-10$ & $Z=\log _{10}\left[2^{1} 2^{0} \theta^{1}(1-\theta)^{0}\right]$ & 0.5 & 0.0 \\
\hline $4-5$ & $Z=\log _{10}\left[2^{1} 2^{0} \theta^{1}(1-\theta)^{0}\right]$ & 0.5 & 0.0 \\
\hline $4-6$ & $Z=\log _{10}\left[2^{1} 2^{0} \theta^{1}(1-\theta)^{0}\right]$ & 0.5 & 0.0 \\
\hline $4-7$ & $Z=\log _{10}\left[2^{1} 2^{0} \theta^{1}(1-\theta)^{0}\right]$ & 0.5 & 0.0 \\
\hline $4-8$ & $Z=\log _{10}\left[2^{1} 2^{0} \theta^{1}(1-\theta)^{0}\right]$ & 0.5 & 0.0 \\
\hline $4-9$ & $Z=\log _{10}\left[2^{1} 2^{0} \theta^{1}(1-\theta)^{0}\right]$ & 0.5 & 0.0 \\
\hline $4-10$ & $Z=\log _{10}\left[2^{1} 2^{0} \theta^{1}(1-\theta)^{0}\right]$ & 0.5 & 0.0 \\
\hline $5-6$ & $Z=\log _{10}\left[2^{1} 2^{0} \theta^{1}(1-\theta)^{0}\right]$ & 0.5 & 0.0 \\
\hline $5-7$ & $Z=\log _{10}\left[2^{0} 2^{1} \theta^{0}(1-\theta)^{1}\right]$ & 0.0 & 0.30 \\
\hline $5-8$ & $Z=\log _{10}\left[2^{1} 2^{0} \theta^{1}(1-\theta)^{0}\right]$ & 0.5 & 0.0 \\
\hline
\end{tabular}




\section{Continued}

$\begin{array}{llll}5-9 & Z=\log _{10}\left[2^{1} 2^{0} \theta^{1}(1-\theta)^{0}\right] & 0.5 & 0.0 \\ 5-10 & Z=\log _{10}\left[2^{0} 2^{1} \theta^{0}(1-\theta)^{1}\right] & 0.0 & 0.30 \\ 6-7 & Z=\log _{10}\left[2^{1} 2^{0} \theta^{1}(1-\theta)^{0}\right] & 0.5 & 0.0 \\ 6-8 & Z=\log _{10}\left[2^{1} 2^{0} \theta^{1}(1-\theta)^{0}\right] & 0.5 & 0.0 \\ 6-9 & Z=\log _{10}\left[2^{1} 2^{0} \theta^{1}(1-\theta)^{0}\right] & 0.5 & 0.0 \\ 6-10 & Z=\log _{10}\left[2^{1} 2^{0} \theta^{1}(1-\theta)^{0}\right] & 0.5 & 0.0 \\ 7-8 & Z=\log _{10}\left[2^{1} 2^{0} \theta^{1}(1-\theta)^{0}\right] & 0.5 & 0.0 \\ 7-9 & Z=\log _{10}\left[2^{1} 2^{0} \theta^{1}(1-\theta)^{0}\right] & 0.5 & 0.0 \\ 7-10 & Z=\log _{10}\left[2^{0} 2^{1} \theta^{0}(1-\theta)^{1}\right] & 0.0 & 0.30 \\ 8-9 & Z=\log _{10}\left[2^{1} 2^{0} \theta^{1}(1-\theta)^{0}\right] & 0.5 & 0.0 \\ 8-10 & Z=\log _{10}\left[2^{1} 2^{0} \theta^{1}(1-\theta)^{0}\right] & 0.5 & 0.0\end{array}$

Table 11. Linkage analysis of family 7.

\begin{tabular}{|c|c|c|c|}
\hline Combination of Markers & LOD & $\theta_{\max }$ & $Z_{\max }^{(7)}$ \\
\hline $1-2$ & $Z=\log _{10}\left[2^{0} 2^{1} \theta^{0}(1-\theta)^{1}\right]$ & 0.0 & 0.30 \\
\hline $1-3$ & $Z=\log _{10}\left[2^{0} 2^{1} \theta^{0}(1-\theta)^{1}\right]$ & 0.0 & 0.30 \\
\hline $1-4$ & $Z=\log _{10}\left[2^{0} 2^{1} \theta^{0}(1-\theta)^{1}\right]$ & 0.0 & 0.30 \\
\hline $1-5$ & $Z=\log _{10}\left[2^{0} 2^{1} \theta^{0}(1-\theta)^{1}\right]$ & 0.0 & 0.30 \\
\hline $1-6$ & $Z=\log _{10}\left[2^{0} 2^{1} \theta^{0}(1-\theta)^{1}\right]$ & 0.0 & 0.30 \\
\hline $1-7$ & $Z=\log _{10}\left[2^{0} 2^{1} \theta^{0}(1-\theta)^{1}\right]$ & 0.0 & 0.30 \\
\hline $1-8$ & $Z=\log _{10}\left[2^{0} 2^{1} \theta^{0}(1-\theta)^{1}\right]$ & 0.0 & 0.30 \\
\hline $1-9$ & $Z=\log _{10}\left[2^{1} 2^{0} \theta^{1}(1-\theta)^{0}\right]$ & 0.5 & 0.0 \\
\hline $1-10$ & $Z=\log _{10}\left[2^{0} 2^{1} \theta^{0}(1-\theta)^{1}\right]$ & 0.0 & 0.30 \\
\hline $2-3$ & $Z=\log _{10}\left[2^{0} 2^{1} \theta^{0}(1-\theta)^{1}\right]$ & 0.0 & 0.30 \\
\hline $2-4$ & $Z=\log _{10}\left[2^{0} 2^{1} \theta^{0}(1-\theta)^{1}\right]$ & 0.0 & 0.30 \\
\hline $2-5$ & $Z=\log _{10}\left[2^{0} 2^{1} \theta^{0}(1-\theta)^{1}\right]$ & 0.0 & 0.30 \\
\hline $2-6$ & $Z=\log _{10}\left[2^{0} 2^{1} \theta^{0}(1-\theta)^{1}\right]$ & 0.0 & 0.30 \\
\hline $2-7$ & $Z=\log _{10}\left[2^{0} 2^{1} \theta^{0}(1-\theta)^{1}\right]$ & 0.0 & 0.30 \\
\hline $2-8$ & $Z=\log _{10}\left[2^{0} 2^{1} \theta^{0}(1-\theta)^{1}\right]$ & 0.0 & 0.30 \\
\hline $2-9$ & $Z=\log _{10}\left[2^{1} 2^{0} \theta^{1}(1-\theta)^{0}\right]$ & 0.5 & 0.0 \\
\hline $2-10$ & $Z=\log _{10}\left[2^{0} 2^{1} \theta^{0}(1-\theta)^{1}\right]$ & 0.0 & 0.30 \\
\hline $3-4$ & $Z=\log _{10}\left[2^{0} 2^{1} \theta^{0}(1-\theta)^{1}\right]$ & 0.0 & 0.30 \\
\hline $3-5$ & $Z=\log _{10}\left[2^{0} 2^{1} \theta^{0}(1-\theta)^{1}\right]$ & 0.0 & 0.30 \\
\hline $3-6$ & $Z=\log _{10}\left[2^{0} 2^{1} \theta^{0}(1-\theta)^{1}\right]$ & 0.0 & 0.30 \\
\hline
\end{tabular}




\section{Continued}

\begin{tabular}{|c|c|c|c|}
\hline $3-7$ & $Z=\log _{10}\left[2^{0} 2^{1} \theta^{0}(1-\theta)^{1}\right]$ & 0.0 & 0.30 \\
\hline $3-8$ & $Z=\log _{10}\left[2^{0} 2^{1} \theta^{0}(1-\theta)^{1}\right]$ & 0.0 & 0.30 \\
\hline $3-9$ & $Z=\log _{10}\left[2^{1} 2^{0} \theta^{1}(1-\theta)^{0}\right]$ & 0.5 & 0.0 \\
\hline $3-10$ & $Z=\log _{10}\left[2^{0} 2^{1} \theta^{0}(1-\theta)^{1}\right]$ & 0.0 & 0.30 \\
\hline $4-5$ & $Z=\log _{10}\left[2^{0} 2^{1} \theta^{0}(1-\theta)^{1}\right]$ & 0.0 & 0.30 \\
\hline $4-6$ & $Z=\log _{10}\left[2^{0} 2^{1} \theta^{0}(1-\theta)^{1}\right]$ & 0.0 & 0.30 \\
\hline $4-7$ & $Z=\log _{10}\left[2^{0} 2^{1} \theta^{0}(1-\theta)^{1}\right]$ & 0.0 & 0.30 \\
\hline $4-8$ & $Z=\log _{10}\left[2^{0} 2^{1} \theta^{0}(1-\theta)^{1}\right]$ & 0.0 & 0.30 \\
\hline $4-9$ & $Z=\log _{10}\left[2^{1} 2^{0} \theta^{1}(1-\theta)^{0}\right]$ & 0.5 & 0.0 \\
\hline $4-10$ & $Z=\log _{10}\left[2^{0} 2^{1} \theta^{0}(1-\theta)^{1}\right]$ & 0.0 & 0.30 \\
\hline $5-6$ & $Z=\log _{10}\left[2^{0} 2^{1} \theta^{0}(1-\theta)^{1}\right]$ & 0.0 & 0.30 \\
\hline $5-7$ & $Z=\log _{10}\left[2^{0} 2^{1} \theta^{0}(1-\theta)^{1}\right]$ & 0.0 & 0.30 \\
\hline $5-8$ & $Z=\log _{10}\left[2^{0} 2^{1} \theta^{0}(1-\theta)^{1}\right]$ & 0.0 & 0.30 \\
\hline $5-9$ & $Z=\log _{10}\left[2^{1} 2^{0} \theta^{1}(1-\theta)^{0}\right]$ & 0.5 & 0.0 \\
\hline $5-10$ & $Z=\log _{10}\left[2^{0} 2^{1} \theta^{0}(1-\theta)^{1}\right]$ & 0.0 & 0.30 \\
\hline $6-7$ & $Z=\log _{10}\left[2^{0} 2^{1} \theta^{0}(1-\theta)^{1}\right]$ & 0.0 & 0.30 \\
\hline $6-8$ & $Z=\log _{10}\left[2^{0} 2^{1} \theta^{0}(1-\theta)^{1}\right]$ & 0.0 & 0.30 \\
\hline $6-9$ & $Z=\log _{10}\left[2^{1} 2^{0} \theta^{1}(1-\theta)^{0}\right]$ & 0.5 & 0.0 \\
\hline $6-10$ & $Z=\log _{10}\left[2^{0} 2^{1} \theta^{0}(1-\theta)^{1}\right]$ & 0.0 & 0.30 \\
\hline $7-8$ & $Z=\log _{10}\left[2^{0} 2^{1} \theta^{0}(1-\theta)^{1}\right]$ & 0.0 & 0.30 \\
\hline $7-9$ & $Z=\log _{10}\left[2^{1} 2^{0} \theta^{1}(1-\theta)^{0}\right]$ & 0.5 & 0.0 \\
\hline $7-10$ & $Z=\log _{10}\left[2^{0} 2^{1} \theta^{0}(1-\theta)^{1}\right]$ & 0.0 & 0.30 \\
\hline $8-9$ & $Z=\log _{10}\left[2^{1} 2^{0} \theta^{1}(1-\theta)^{0}\right]$ & 0.5 & 0.0 \\
\hline $8-10$ & $Z=\log _{10}\left[2^{0} 2^{1} \theta^{0}(1-\theta)^{1}\right]$ & 0.0 & 0.30 \\
\hline
\end{tabular}

Table 12. Linkage analysis of family 8.

\begin{tabular}{cccc}
\hline Combination of Markers & LOD & $\theta_{\text {max }}$ & $Z_{\text {max }}^{(8)}$ \\
\hline $1-2$ & $Z=\log _{10}\left[2^{1} 2^{1} \theta^{1}(1-\theta)^{1}\right]$ & 0.5 & 0.0 \\
$1-3$ & $Z=\log _{10}\left[2^{2} 2^{0} \theta^{2}(1-\theta)^{0}\right]$ & 0.5 & 0.0 \\
$1-4$ & $Z=\log _{10}\left[2^{2} 2^{0} \theta^{2}(1-\theta)^{0}\right]$ & 0.5 & 0.0 \\
$1-5$ & $Z=\log _{10}\left[2^{1} 2^{1} \theta^{1}(1-\theta)^{1}\right]$ & 0.5 & 0.0 \\
$1-6$ & $Z=\log _{10}\left[2^{2} 2^{0} \theta^{2}(1-\theta)^{0}\right]$ & 0.5 & 0.0 \\
$1-7$ & $Z=\log _{10}\left[2^{1} 2^{1} \theta^{1}(1-\theta)^{1}\right]$ & 0.5 & 0.0 \\
$1-8$ & $Z=\log _{10}\left[2^{2} 2^{0} \theta^{2}(1-\theta)^{0}\right]$ & 0.5 & 0.0
\end{tabular}




\section{Continued}

\begin{tabular}{|c|c|c|c|}
\hline $1-10$ & $Z=\log _{10}\left[2^{1} 2^{1} \theta^{1}(1-\theta)^{1}\right]$ & 0.5 & 0.0 \\
\hline $2-3$ & $Z=\log _{10}\left[2^{2} 2^{0} \theta^{2}(1-\theta)^{0}\right]$ & 0.5 & 0.0 \\
\hline $2-4$ & $Z=\log _{10}\left[2^{2} 2^{0} \theta^{2}(1-\theta)^{0}\right]$ & 0.5 & 0.0 \\
\hline $2-5$ & $Z=\log _{10}\left[2^{0} 2^{2} \theta^{0}(1-\theta)^{2}\right]$ & 0.0 & 0.60 \\
\hline $2-6$ & $Z=\log _{10}\left[2^{2} 2^{0} \theta^{2}(1-\theta)^{0}\right]$ & 0.5 & 0.0 \\
\hline $2-7$ & $Z=\log _{10}\left[2^{0} 2^{2} \theta^{0}(1-\theta)^{2}\right]$ & 0.0 & 0.60 \\
\hline $2-8$ & $Z=\log _{10}\left[2^{2} 2^{0} \theta^{2}(1-\theta)^{0}\right]$ & 0.5 & 0.0 \\
\hline $2-9$ & $Z=\log _{10}\left[2^{0} 2^{2} \theta^{0}(1-\theta)^{2}\right]$ & 0.0 & 0.60 \\
\hline $2-10$ & $Z=\log _{10}\left[2^{1} 2^{1} \theta^{1}(1-\theta)^{1}\right]$ & 0.0 & 0.0 \\
\hline $3-4$ & $Z=\log _{10}\left[2^{2} 2^{0} \theta^{2}(1-\theta)^{0}\right]$ & 0.5 & 0.0 \\
\hline $3-5$ & $Z=\log _{10}\left[2^{2} 2^{0} \theta^{2}(1-\theta)^{0}\right]$ & 0.5 & 0.0 \\
\hline $3-6$ & $Z=\log _{10}\left[2^{2} 2^{0} \theta^{2}(1-\theta)^{0}\right]$ & 0.5 & 0.0 \\
\hline $3-7$ & $Z=\log _{10}\left[2^{2} 2^{0} \theta^{2}(1-\theta)^{0}\right]$ & 0.5 & 0.0 \\
\hline $3-8$ & $Z=\log _{10}\left[2^{2} 2^{0} \theta^{2}(1-\theta)^{0}\right]$ & 0.5 & 0.0 \\
\hline $3-9$ & $Z=\log _{10}\left[2^{2} 2^{0} \theta^{2}(1-\theta)^{0}\right]$ & 0.5 & 0.0 \\
\hline $3-10$ & $Z=\log _{10}\left[2^{2} 2^{0} \theta^{2}(1-\theta)^{0}\right]$ & 0.5 & 0.0 \\
\hline $4-5$ & $Z=\log _{10}\left[2^{2} 2^{0} \theta^{2}(1-\theta)^{0}\right]$ & 0.5 & 0.0 \\
\hline $4-6$ & $Z=\log _{10}\left[2^{2} 2^{0} \theta^{2}(1-\theta)^{0}\right]$ & 0.5 & 0.0 \\
\hline $4-7$ & $Z=\log _{10}\left[2^{2} 2^{0} \theta^{2}(1-\theta)^{0}\right]$ & 0.5 & 0.0 \\
\hline $4-8$ & $Z=\log _{10}\left[2^{2} 2^{0} \theta^{2}(1-\theta)^{0}\right]$ & 0.5 & 0.0 \\
\hline $4-9$ & $Z=\log _{10}\left[2^{2} 2^{0} \theta^{2}(1-\theta)^{0}\right]$ & 0.5 & 0.0 \\
\hline $4-10$ & $Z=\log _{10}\left[2^{2} 2^{0} \theta^{2}(1-\theta)^{0}\right]$ & 0.5 & 0.0 \\
\hline $5-6$ & $Z=\log _{10}\left[2^{2} 2^{0} \theta^{2}(1-\theta)^{0}\right]$ & 0.5 & 0.0 \\
\hline $5-7$ & $Z=\log _{10}\left[2^{0} 2^{2} \theta^{0}(1-\theta)^{2}\right]$ & 0.0 & 0.60 \\
\hline $5-8$ & $Z=\log _{10}\left[2^{2} 2^{0} \theta^{2}(1-\theta)^{0}\right]$ & 0.5 & 0.0 \\
\hline $5-9$ & $Z=\log _{10}\left[2^{0} 2^{2} \theta^{0}(1-\theta)^{2}\right]$ & 0.0 & 0.60 \\
\hline $5-10$ & $Z=\log _{10}\left[2^{1} 2^{1} \theta^{1}(1-\theta)^{1}\right]$ & 0.5 & 0.0 \\
\hline $6-7$ & $Z=\log _{10}\left[2^{2} 2^{0} \theta^{2}(1-\theta)^{0}\right]$ & 0.5 & 0.0 \\
\hline $6-8$ & $Z=\log _{10}\left[2^{2} 2^{0} \theta^{2}(1-\theta)^{0}\right]$ & 0.5 & 0.0 \\
\hline $6-9$ & $Z=\log _{10}\left[2^{2} 2^{0} \theta^{2}(1-\theta)^{0}\right]$ & 0.5 & 0.0 \\
\hline $6-10$ & $Z=\log _{10}\left[2^{2} 2^{0} \theta^{2}(1-\theta)^{0}\right]$ & 0.5 & 0.0 \\
\hline $7-8$ & $Z=\log _{10}\left[2^{2} 2^{0} \theta^{2}(1-\theta)^{0}\right]$ & 0.5 & 0.0 \\
\hline $7-9$ & $Z=\log _{10}\left[2^{0} 2^{2} \theta^{0}(1-\theta)^{2}\right]$ & 0.0 & 0.60 \\
\hline $7-10$ & $Z=\log _{10}\left[2^{1} 2^{1} \theta^{1}(1-\theta)^{1}\right]$ & 0.5 & 0.0 \\
\hline
\end{tabular}




\section{Continued}

$\begin{array}{llll}8-9 & Z=\log _{10}\left[2^{2} 2^{0} \theta^{2}(1-\theta)^{0}\right] & 0.5 & 0.0 \\ 8-10 & Z=\log _{10}\left[2^{2} 2^{0} \theta^{2}(1-\theta)^{0}\right] & 0.5 & 0.0\end{array}$

Table 13. Linkage analysis of family 9.

\begin{tabular}{|c|c|c|c|}
\hline Combination of Markers & LOD & $\theta_{\max }$ & $Z_{\max }^{(9)}$ \\
\hline $1-2$ & $Z=\log _{10}\left[2^{1} 2^{2} \theta^{1}(1-\theta)^{2}\right]$ & 0.33 & 0.07 \\
\hline $1-3$ & $Z=\log _{10}\left[2^{3} 2^{0} \theta^{3}(1-\theta)^{0}\right]$ & 0.5 & 0.0 \\
\hline $1-4$ & $Z=\log _{10}\left[2^{3} 2^{0} \theta^{3}(1-\theta)^{0}\right]$ & 0.5 & 0.0 \\
\hline $1-5$ & $Z=\log _{10}\left[2^{3} 2^{0} \theta^{3}(1-\theta)^{0}\right]$ & 0.5 & 0.0 \\
\hline $1-6$ & $Z=\log _{10}\left[2^{3} 2^{0} \theta^{3}(1-\theta)^{0}\right]$ & 0.5 & 0.0 \\
\hline $1-7$ & $Z=\log _{10}\left[2^{0} 2^{3} \theta^{0}(1-\theta)^{3}\right]$ & 0.0 & 0.90 \\
\hline $1-8$ & $Z=\log _{10}\left[2^{3} 2^{0} \theta^{3}(1-\theta)^{0}\right]$ & 0.5 & 0.0 \\
\hline $1-9$ & $Z=\log _{10}\left[2^{3} 2^{0} \theta^{3}(1-\theta)^{0}\right]$ & 0.5 & 0.0 \\
\hline $1-10$ & $Z=\log _{10}\left[2^{2} 2^{1} \theta^{2}(1-\theta)^{1}\right]$ & 0.5 & 0.0 \\
\hline $2-3$ & $Z=\log _{10}\left[2^{3} 2^{0} \theta^{3}(1-\theta)^{0}\right]$ & 0.5 & 0.0 \\
\hline $2-4$ & $Z=\log _{10}\left[2^{3} 2^{0} \theta^{3}(1-\theta)^{0}\right]$ & 0.5 & 0.0 \\
\hline $2-5$ & $Z=\log _{10}\left[2^{3} 2^{2} \theta^{3}(1-\theta)^{0}\right]$ & 0.5 & 0.0 \\
\hline $2-6$ & $Z=\log _{10}\left[2^{1} 2^{2} \theta^{1}(1-\theta)^{2}\right]$ & 0.33 & 0.07 \\
\hline $2-7$ & $Z=\log _{10}\left[2^{1} 2^{2} \theta^{1}(1-\theta)^{2}\right]$ & 0.33 & 0.07 \\
\hline $2-8$ & $Z=\log _{10}\left[2^{3} 2^{0} \theta^{3}(1-\theta)^{0}\right]$ & 0.5 & 0.0 \\
\hline $2-9$ & $Z=\log _{10}\left[2^{3} 2^{0} \theta^{3}(1-\theta)^{0}\right]$ & 0.5 & 0.0 \\
\hline $2-10$ & $Z=\log _{10}\left[2^{3} 2^{0} \theta^{3}(1-\theta)^{0}\right]$ & 0.5 & 0.0 \\
\hline $3-4$ & $Z=\log _{10}\left[2^{3} 2^{0} \theta^{3}(1-\theta)^{0}\right]$ & 0.5 & 0.0 \\
\hline $3-5$ & $Z=\log _{10}\left[2^{3} 2^{0} \theta^{3}(1-\theta)^{0}\right]$ & 0.5 & 0.0 \\
\hline $3-6$ & $Z=\log _{10}\left[2^{3} 2^{0} \theta^{3}(1-\theta)^{0}\right]$ & 0.5 & 0.0 \\
\hline $3-7$ & $Z=\log _{10}\left[2^{3} 2^{0} \theta^{3}(1-\theta)^{0}\right]$ & 0.5 & 0.0 \\
\hline $3-8$ & $Z=\log _{10}\left[2^{3} 2^{0} \theta^{3}(1-\theta)^{0}\right]$ & 0.5 & 0.0 \\
\hline $3-9$ & $Z=\log _{10}\left[2^{2} 2^{1} \theta^{2}(1-\theta)^{1}\right]$ & 0.5 & 0.0 \\
\hline $3-10$ & $Z=\log _{10}\left[2^{2} 2^{1} \theta^{2}(1-\theta)^{1}\right]$ & 0.5 & 0.0 \\
\hline $4-5$ & $Z=\log _{10}\left[2^{3} 2^{0} \theta^{3}(1-\theta)^{0}\right]$ & 0.5 & 0.0 \\
\hline $4-6$ & $Z=\log _{10}\left[2^{3} 2^{0} \theta^{3}(1-\theta)^{0}\right]$ & 0.5 & 0.0 \\
\hline $4-7$ & $Z=\log _{10}\left[2^{3} 2^{0} \theta^{3}(1-\theta)^{3}\right]$ & 0.0 & 0.9 \\
\hline $4-8$ & $Z=\log _{10}\left[2^{3} 2^{0} \theta^{3}(1-\theta)^{0}\right]$ & 0.5 & 0.0 \\
\hline $4-9$ & $Z=\log _{10}\left[2^{3} 2^{0} \theta^{3}(1-\theta)^{0}\right]$ & 0.5 & 0.0 \\
\hline $4-10$ & $Z=\log _{10}\left[2^{3} 2^{0} \theta^{3}(1-\theta)^{0}\right]$ & 0.5 & 0.0 \\
\hline
\end{tabular}




\section{Continued}

$\begin{array}{llll}5-6 & Z=\log _{10}\left[2^{3} 2^{0} \theta^{3}(1-\theta)^{0}\right] & 0.5 & 0.0 \\ 5-7 & Z=\log _{10}\left[2^{3} 2^{0} \theta^{3}(1-\theta)^{0}\right] & 0.5 & 0.0 \\ 5-8 & Z=\log _{10}\left[2^{3} 2^{0} \theta^{3}(1-\theta)^{0}\right] & 0.5 & 0.0 \\ 5-9 & Z=\log _{10}\left[2^{3} 2^{0} \theta^{3}(1-\theta)^{0}\right] & 0.5 & 0.0 \\ 5-10 & Z=\log _{10}\left[2^{3} 2^{0} \theta^{3}(1-\theta)^{0}\right] & 0.5 & 0.0 \\ 6-7 & Z=\log _{10}\left[2^{3} 2^{0} \theta^{3}(1-\theta)^{0}\right] & 0.5 & 0.0 \\ 6-8 & Z=\log _{10}\left[2^{3} 2^{0} \theta^{3}(1-\theta)^{0}\right] & 0.5 & 0.0 \\ 6-9 & Z=\log _{10}\left[2^{3} 2^{0} \theta^{3}(1-\theta)^{0}\right] & 0.5 & 0.0 \\ 6-10 & Z=\log _{10}\left[2^{3} 2^{0} \theta^{3}(1-\theta)^{0}\right] & 0.5 & 0.0 \\ 7-8 & Z=\log _{10}\left[2^{3} 2^{0} \theta^{3}(1-\theta)^{0}\right] & 0.5 & 0.0 \\ 7-9 & Z=\log _{10}\left[2^{3} 2^{0} \theta^{3}(1-\theta)^{0}\right] & 0.5 & 0.5 \\ 7-10 & Z=\log _{10}\left[2^{3} 2^{0} \theta^{3}(1-\theta)^{0}\right] & 0.5 & 0.0 \\ 8-9 & Z=\log _{10}\left[2^{3} 2^{0} \theta^{3}(1-\theta)^{0}\right] & 0.5 & 0.0 \\ 8-10 & Z=\log _{10}\left[2^{3} 2^{0} \theta^{3}(1-\theta)^{0}\right] & 0.5 & 0.0\end{array}$

Table 14. Linkage analysis of family 10.

\begin{tabular}{|c|c|c|c|}
\hline Combination of Markers & LOD & $\theta_{\max }$ & $Z_{\max }^{(10)}$ \\
\hline $1-2$ & $Z=\log _{10}\left[2^{0} 2^{1} \theta^{0}(1-\theta)^{1}\right]$ & 0.0 & 0.30 \\
\hline $1-3$ & $Z=\log _{10}\left[2^{0} 2^{1} \theta^{0}(1-\theta)^{1}\right]$ & 0.0 & 0.30 \\
\hline $1-4$ & $Z=\log _{10}\left[2^{0} 2^{1} \theta^{0}(1-\theta)^{1}\right]$ & 0.0 & 0.30 \\
\hline $1-5$ & $Z=\log _{10}\left[2^{0} 2^{1} \theta^{0}(1-\theta)^{1}\right]$ & 0.0 & 0.30 \\
\hline $1-6$ & $Z=\log _{10}\left[2^{0} 2^{1} \theta^{0}(1-\theta)^{1}\right]$ & 0.0 & 0.30 \\
\hline $1-7$ & $Z=\log _{10}\left[2^{0} 2^{1} \theta^{0}(1-\theta)^{1}\right]$ & 0.0 & 0.30 \\
\hline $1-8$ & $Z=\log _{10}\left[2^{0} 2^{1} \theta^{0}(1-\theta)^{1}\right]$ & 0.0 & 0.30 \\
\hline $1-9$ & $Z=\log _{10}\left[2^{0} 2^{1} \theta^{0}(1-\theta)^{1}\right]$ & 0.0 & 0.30 \\
\hline $1-10$ & $Z=\log _{10}\left[2^{0} 2^{1} \theta^{0}(1-\theta)^{1}\right]$ & 0.0 & 0.30 \\
\hline $2-3$ & $Z=\log _{10}\left[2^{0} 2^{1} \theta^{0}(1-\theta)^{1}\right]$ & 0.0 & 0.30 \\
\hline $2-4$ & $Z=\log _{10}\left[2^{0} 2^{1} \theta^{0}(1-\theta)^{1}\right]$ & 0.0 & 0.30 \\
\hline $2-5$ & $Z=\log _{10}\left[2^{0} 2^{1} \theta^{0}(1-\theta)^{1}\right]$ & 0.0 & 0.30 \\
\hline $2-6$ & $Z=\log _{10}\left[2^{0} 2^{1} \theta^{0}(1-\theta)^{1}\right]$ & 0.0 & 0.30 \\
\hline $2-7$ & $Z=\log _{10}\left[2^{0} 2^{1} \theta^{0}(1-\theta)^{1}\right]$ & 0.0 & 0.30 \\
\hline $2-8$ & $Z=\log _{10}\left[2^{0} 2^{1} \theta^{0}(1-\theta)^{1}\right]$ & 0.0 & 0.30 \\
\hline $2-9$ & $Z=\log _{10}\left[2^{0} 2^{1} \theta^{0}(1-\theta)^{1}\right]$ & 0.0 & 0.30 \\
\hline $2-10$ & $Z=\log _{10}\left[2^{0} 2^{1} \theta^{0}(1-\theta)^{1}\right]$ & 0.0 & 0.30 \\
\hline $3-4$ & $Z=\log _{10}\left[2^{0} 2^{1} \theta^{0}(1-\theta)^{1}\right]$ & 0.0 & 0.30 \\
\hline
\end{tabular}




\section{Continued}

\begin{tabular}{|c|c|c|c|}
\hline $3-5$ & $Z=\log _{10}\left[2^{0} 2^{1} \theta^{0}(1-\theta)^{1}\right]$ & 0.0 & 0.30 \\
\hline $3-6$ & $Z=\log _{10}\left[2^{0} 2^{1} \theta^{0}(1-\theta)^{1}\right]$ & 0.0 & 0.30 \\
\hline $3-7$ & $Z=\log _{10}\left[2^{0} 2^{1} \theta^{0}(1-\theta)^{1}\right]$ & 0.0 & 0.30 \\
\hline $3-8$ & $Z=\log _{10}\left[2^{0} 2^{1} \theta^{0}(1-\theta)^{1}\right]$ & 0.0 & 0.30 \\
\hline $3-9$ & $Z=\log _{10}\left[2^{0} 2^{1} \theta^{0}(1-\theta)^{1}\right]$ & 0.0 & 0.30 \\
\hline $3-10$ & $Z=\log _{10}\left[2^{0} 2^{1} \theta^{0}(1-\theta)^{1}\right]$ & 0.0 & 0.30 \\
\hline $4-5$ & $Z=\log _{10}\left[2^{0} 2^{1} \theta^{0}(1-\theta)^{1}\right]$ & 0.0 & 0.30 \\
\hline $4-6$ & $Z=\log _{10}\left[2^{0} 2^{1} \theta^{0}(1-\theta)^{1}\right]$ & 0.0 & 0.30 \\
\hline $4-7$ & $Z=\log _{10}\left[2^{0} 2^{1} \theta^{0}(1-\theta)^{1}\right]$ & 0.0 & 0.30 \\
\hline $4-8$ & $Z=\log _{10}\left[2^{0} 2^{1} \theta^{0}(1-\theta)^{1}\right]$ & 0.0 & 0.30 \\
\hline $4-9$ & $Z=\log _{10}\left[2^{0} 2^{1} \theta^{0}(1-\theta)^{1}\right]$ & 0.0 & 0.30 \\
\hline $4-10$ & $Z=\log _{10}\left[2^{0} 2^{1} \theta^{0}(1-\theta)^{1}\right]$ & 0.0 & 0.30 \\
\hline $5-6$ & $Z=\log _{10}\left[2^{0} 2^{1} \theta^{0}(1-\theta)^{1}\right]$ & 0.0 & 0.30 \\
\hline $5-7$ & $Z=\log _{10}\left[2^{0} 2^{1} \theta^{0}(1-\theta)^{1}\right]$ & 0.0 & 0.30 \\
\hline $5-8$ & $Z=\log _{10}\left[2^{0} 2^{1} \theta^{0}(1-\theta)^{1}\right]$ & 0.0 & 0.30 \\
\hline $5-9$ & $Z=\log _{10}\left[2^{0} 2^{1} \theta^{0}(1-\theta)^{1}\right]$ & 0.0 & 0.30 \\
\hline $5-10$ & $Z=\log _{10}\left[2^{0} 2^{1} \theta^{0}(1-\theta)^{1}\right]$ & 0.0 & 0.30 \\
\hline $6-7$ & $Z=\log _{10}\left[2^{1} 2^{0} \theta^{1}(1-\theta)^{0}\right]$ & 0.5 & 0.0 \\
\hline $6-8$ & $Z=\log _{10}\left[2^{1} 2^{0} \theta^{1}(1-\theta)^{0}\right]$ & 0.5 & 0.0 \\
\hline $6-9$ & $Z=\log _{10}\left[2^{1} 2^{0} \theta^{1}(1-\theta)^{0}\right]$ & 0.5 & 0.0 \\
\hline $6-10$ & $Z=\log _{10}\left[2^{1} 2^{0} \theta^{1}(1-\theta)^{0}\right]$ & 0.5 & 0.0 \\
\hline $7-8$ & $Z=\log _{10}\left[2^{0} 2^{1} \theta^{0}(1-\theta)^{1}\right]$ & 0.0 & 0.30 \\
\hline $7-9$ & $Z=\log _{10}\left[2^{0} 2^{1} \theta^{0}(1-\theta)^{1}\right]$ & 0.0 & 0.30 \\
\hline $7-10$ & $Z=\log _{10}\left[2^{0} 2^{1} \theta^{0}(1-\theta)^{1}\right]$ & 0.0 & 0.30 \\
\hline $8-9$ & $Z=\log _{10}\left[2^{0} 2^{1} \theta^{0}(1-\theta)^{1}\right]$ & 0.0 & 0.30 \\
\hline $8-10$ & $Z=\log _{10}\left[2^{0} 2^{1} \theta^{0}(1-\theta)^{1}\right]$ & 0.0 & 0.30 \\
\hline
\end{tabular}

Table 15. Linkage analysis of family 11.

\begin{tabular}{cccc}
\hline Combination of Markers & LOD & $\theta_{\max }$ & $Z_{\max }^{(11)}$ \\
\hline $1-2$ & $Z=\log _{10}\left[2^{0} 2^{1} \theta^{0}(1-\theta)^{1}\right]$ & 0.0 & 0.30 \\
$1-3$ & $Z=\log _{10}\left[2^{1} 2^{0} \theta^{1}(1-\theta)^{0}\right]$ & 0.5 & 0.0 \\
$1-4$ & $Z=\log _{10}\left[2^{1} 2^{0} \theta^{1}(1-\theta)^{0}\right]$ & 0.5 & 0.0 \\
$1-5$ & $Z=\log _{10}\left[2^{1} 2^{0} \theta^{1}(1-\theta)^{0}\right]$ & 0.5 & 0.0 \\
$1-6$ & $Z=\log _{10}\left[2^{1} 2^{0} \theta^{1}(1-\theta)^{0}\right]$ & 0.5 & 0.0 \\
$1-7$ & $Z=\log _{10}\left[2^{1} 2^{0} \theta^{1}(1-\theta)^{0}\right]$ & 0.5 & 0.0 \\
\hline
\end{tabular}




\section{Continued}

\begin{tabular}{|c|c|c|c|}
\hline $1-8$ & $Z=\log _{10}\left[2^{0} 2^{1} \theta^{0}(1-\theta)^{1}\right]$ & 0.0 & 0.30 \\
\hline $1-9$ & $Z=\log _{10}\left[2^{0} 2^{1} \theta^{0}(1-\theta)^{1}\right]$ & 0.0 & 0.30 \\
\hline $1-10$ & $Z=\log _{10}\left[2^{0} 2^{1} \theta^{0}(1-\theta)^{1}\right]$ & 0.0 & 0.30 \\
\hline $2-3$ & $Z=\log _{10}\left[2^{1} 2^{0} \theta^{1}(1-\theta)^{0}\right]$ & 0.5 & 0.0 \\
\hline $2-4$ & $Z=\log _{10}\left[2^{1} 2^{0} \theta^{1}(1-\theta)^{0}\right]$ & 0.5 & 0.0 \\
\hline $2-5$ & $Z=\log _{10}\left[2^{1} 2^{0} \theta^{1}(1-\theta)^{0}\right]$ & 0.5 & 0.0 \\
\hline $2-6$ & $Z=\log _{10}\left[2^{1} 2^{0} \theta^{1}(1-\theta)^{0}\right]$ & 0.5 & 0.0 \\
\hline $2-7$ & $Z=\log _{10}\left[2^{1} 2^{0} \theta^{1}(1-\theta)^{0}\right]$ & 0.5 & 0.0 \\
\hline $2-8$ & $Z=\log _{10}\left[2^{0} 2^{1} \theta^{0}(1-\theta)^{1}\right]$ & 0.0 & 0.30 \\
\hline $2-9$ & $Z=\log _{10}\left[2^{0} 2^{1} \theta^{0}(1-\theta)^{1}\right]$ & 0.0 & 0.30 \\
\hline $2-10$ & $Z=\log _{10}\left[2^{0} 2^{1} \theta^{0}(1-\theta)^{1}\right]$ & 0.0 & 0.30 \\
\hline $3-4$ & $Z=\log _{10}\left[2^{1} 2^{0} \theta^{1}(1-\theta)^{0}\right]$ & 0.5 & 0.0 \\
\hline $3-5$ & $Z=\log _{10}\left[2^{1} 2^{0} \theta^{1}(1-\theta)^{0}\right]$ & 0.5 & 0.0 \\
\hline $3-6$ & $Z=\log _{10}\left[2^{1} 2^{0} \theta^{1}(1-\theta)^{0}\right]$ & 0.5 & 0.0 \\
\hline $3-7$ & $Z=\log _{10}\left[2^{1} 2^{0} \theta^{1}(1-\theta)^{0}\right]$ & 0.5 & 0.0 \\
\hline $3-8$ & $Z=\log _{10}\left[2^{1} 2^{0} \theta^{1}(1-\theta)^{0}\right]$ & 0.5 & 0.0 \\
\hline $3-9$ & $Z=\log _{10}\left[2^{1} 2^{0} \theta^{1}(1-\theta)^{0}\right]$ & 0.5 & 0.0 \\
\hline $3-10$ & $Z=\log _{10}\left[2^{1} 2^{0} \theta^{1}(1-\theta)^{0}\right]$ & 0.5 & 0.0 \\
\hline $4-5$ & $Z=\log _{10}\left[2^{1} 2^{0} \theta^{1}(1-\theta)^{0}\right]$ & 0.5 & 0.0 \\
\hline $4-6$ & $Z=\log _{10}\left[2^{1} 2^{0} \theta^{1}(1-\theta)^{0}\right]$ & 0.5 & 0.0 \\
\hline $4-7$ & $Z=\log _{10}\left[2^{1} 2^{0} \theta^{1}(1-\theta)^{0}\right]$ & 0.5 & 0.0 \\
\hline $4-8$ & $Z=\log _{10}\left[2^{1} 2^{0} \theta^{1}(1-\theta)^{0}\right]$ & 0.5 & 0.0 \\
\hline $4-9$ & $Z=\log _{10}\left[2^{1} 2^{0} \theta^{1}(1-\theta)^{0}\right]$ & 0.5 & 0.0 \\
\hline $4-10$ & $Z=\log _{10}\left[2^{1} 2^{0} \theta^{1}(1-\theta)^{0}\right]$ & 0.5 & 0.0 \\
\hline $5-6$ & $Z=\log _{10}\left[2^{1} 2^{0} \theta^{1}(1-\theta)^{0}\right]$ & 0.5 & 0.0 \\
\hline $5-7$ & $Z=\log _{10}\left[2^{1} 2^{0} \theta^{1}(1-\theta)^{0}\right]$ & 0.5 & 0.0 \\
\hline $5-8$ & $Z=\log _{10}\left[2^{1} 2^{0} \theta^{1}(1-\theta)^{0}\right]$ & 0.5 & 0.0 \\
\hline $5-9$ & $Z=\log _{10}\left[2^{1} 2^{0} \theta^{1}(1-\theta)^{0}\right]$ & 0.5 & 0.0 \\
\hline $5-10$ & $Z=\log _{10}\left[2^{1} 2^{0} \theta^{1}(1-\theta)^{0}\right]$ & 0.5 & 0.0 \\
\hline $6-7$ & $Z=\log _{10}\left[2^{1} 2^{0} \theta^{1}(1-\theta)^{0}\right]$ & 0.5 & 0.0 \\
\hline $6-8$ & $Z=\log _{10}\left[2^{1} 2^{0} \theta^{1}(1-\theta)^{0}\right]$ & 0.5 & 0.0 \\
\hline $6-9$ & $Z=\log _{10}\left[2^{1} 2^{0} \theta^{1}(1-\theta)^{0}\right]$ & 0.5 & 0.0 \\
\hline $6-10$ & $Z=\log _{10}\left[2^{1} 2^{0} \theta^{1}(1-\theta)^{0}\right]$ & 0.5 & 0.0 \\
\hline $7-8$ & $Z=\log _{10}\left[2^{1} 2^{0} \theta^{1}(1-\theta)^{0}\right]$ & 0.5 & 0.0 \\
\hline
\end{tabular}




\section{Continued}

$\begin{array}{llll}7-9 & Z=\log _{10}\left[2^{1} 2^{0} \theta^{1}(1-\theta)^{0}\right] & 0.5 & 0.0 \\ 7-10 & Z=\log _{10}\left[2^{1} 2^{0} \theta^{1}(1-\theta)^{0}\right] & 0.5 & 0.0 \\ 8-9 & Z=\log _{10}\left[2^{0} 2^{1} \theta^{0}(1-\theta)^{1}\right] & 0.0 & 0.30 \\ 8-10 & Z=\log _{10}\left[2^{0} 2^{1} \theta^{0}(1-\theta)^{1}\right] & 0.0 & 0.30\end{array}$

Table 16. Linkage analysis of family 12.

\begin{tabular}{|c|c|c|c|}
\hline Combination of Markers & LOD & $\theta_{\max }$ & $Z_{\max }^{(12)}$ \\
\hline $1-2$ & $Z=\log _{10}\left[2^{1} 2^{0} \theta^{1}(1-\theta)^{0}\right]$ & 0.5 & 0.0 \\
\hline $1-3$ & $Z=\log _{10}\left[2^{1} 2^{0} \theta^{1}(1-\theta)^{0}\right]$ & 0.5 & 0.0 \\
\hline $1-4$ & $Z=\log _{10}\left[2^{1} 2^{0} \theta^{1}(1-\theta)^{0}\right]$ & 0.5 & 0.0 \\
\hline $1-5$ & $Z=\log _{10}\left[2^{1} 2^{0} \theta^{1}(1-\theta)^{0}\right]$ & 0.5 & 0.0 \\
\hline $1-6$ & $Z=\log _{10}\left[2^{1} 2^{0} \theta^{1}(1-\theta)^{0}\right]$ & 0.5 & 0.0 \\
\hline $1-7$ & $Z=\log _{10}\left[2^{1} 2^{0} \theta^{1}(1-\theta)^{0}\right]$ & 0.5 & 0.0 \\
\hline $1-8$ & $Z=\log _{10}\left[2^{1} 2^{0} \theta^{1}(1-\theta)^{0}\right]$ & 0.5 & 0.0 \\
\hline $1-9$ & $Z=\log _{10}\left[2^{1} 2^{0} \theta^{1}(1-\theta)^{0}\right]$ & 0.5 & 0.0 \\
\hline $1-10$ & $Z=\log _{10}\left[2^{1} 2^{0} \theta^{1}(1-\theta)^{0}\right]$ & 0.5 & 0.0 \\
\hline $2-3$ & $Z=\log _{10}\left[2^{1} 2^{0} \theta^{1}(1-\theta)^{0}\right]$ & 0.5 & 0.0 \\
\hline $2-4$ & $Z=\log _{10}\left[2^{1} 2^{0} \theta^{1}(1-\theta)^{0}\right]$ & 0.5 & 0.0 \\
\hline $2-5$ & $Z=\log _{10}\left[2^{1} 2^{0} \theta^{1}(1-\theta)^{0}\right]$ & 0.5 & 0.0 \\
\hline $2-6$ & $Z=\log _{10}\left[2^{1} 2^{0} \theta^{1}(1-\theta)^{0}\right]$ & 0.5 & 0.0 \\
\hline $2-7$ & $Z=\log _{10}\left[2^{1} 2^{0} \theta^{1}(1-\theta)^{0}\right]$ & 0.5 & 0.0 \\
\hline $2-8$ & $Z=\log _{10}\left[2^{1} 2^{0} \theta^{1}(1-\theta)^{0}\right]$ & 0.5 & 0.0 \\
\hline $2-9$ & $Z=\log _{10}\left[2^{1} 2^{0} \theta^{1}(1-\theta)^{0}\right]$ & 0.5 & 0.0 \\
\hline $2-10$ & $Z=\log _{10}\left[2^{1} 2^{0} \theta^{1}(1-\theta)^{0}\right]$ & 0.5 & 0.0 \\
\hline $3-4$ & $Z=\log _{10}\left[2^{0} 2^{1} \theta^{0}(1-\theta)^{1}\right]$ & 0.0 & 0.30 \\
\hline $3-5$ & $Z=\log _{10}\left[2^{0} 2^{1} \theta^{0}(1-\theta)^{1}\right]$ & 0.0 & 0.30 \\
\hline $3-6$ & $Z=\log _{10}\left[2^{0} 2^{1} \theta^{0}(1-\theta)^{1}\right]$ & 0.0 & 0.30 \\
\hline $3-7$ & $Z=\log _{10}\left[2^{0} 2^{1} \theta^{0}(1-\theta)^{1}\right]$ & 0.0 & 0.30 \\
\hline $3-8$ & $Z=\log _{10}\left[2^{0} 2^{1} \theta^{0}(1-\theta)^{1}\right]$ & 0.0 & 0.30 \\
\hline $3-9$ & $Z=\log _{10}\left[2^{1} 2^{0} \theta^{1}(1-\theta)^{0}\right]$ & 0.5 & 0.0 \\
\hline $3-10$ & $Z=\log _{10}\left[2^{0} 2^{1} \theta^{0}(1-\theta)^{1}\right]$ & 0.0 & 0.30 \\
\hline $4-5$ & $Z=\log _{10}\left[2^{0} 2^{1} \theta^{0}(1-\theta)^{1}\right]$ & 0.0 & 0.30 \\
\hline
\end{tabular}




\section{Continued}

\begin{tabular}{|c|c|c|c|}
\hline $4-6$ & $Z=\log _{10}\left[2^{0} 2^{1} \theta^{0}(1-\theta)^{1}\right]$ & 0.0 & 0.30 \\
\hline $4-7$ & $Z=\log _{10}\left[2^{0} 2^{1} \theta^{0}(1-\theta)^{1}\right]$ & 0.0 & 0.30 \\
\hline $4-8$ & $Z=\log _{10}\left[2^{0} 2^{1} \theta^{0}(1-\theta)^{1}\right]$ & 0.0 & 0.30 \\
\hline $4-9$ & $Z=\log _{10}\left[2^{1} 2^{0} \theta^{1}(1-\theta)^{0}\right]$ & 0.5 & 0.0 \\
\hline $4-10$ & $Z=\log _{10}\left[2^{0} 2^{1} \theta^{0}(1-\theta)^{1}\right]$ & 0.0 & 0.30 \\
\hline $5-6$ & $Z=\log _{10}\left[2^{0} 2^{1} \theta^{0}(1-\theta)^{1}\right]$ & 0.0 & 0.30 \\
\hline $5-7$ & $Z=\log _{10}\left[2^{0} 2^{1} \theta^{0}(1-\theta)^{1}\right]$ & 0.0 & 0.30 \\
\hline $5-8$ & $Z=\log _{10}\left[2^{0} 2^{1} \theta^{0}(1-\theta)^{1}\right]$ & 0.0 & 0.30 \\
\hline $5-9$ & $Z=\log _{10}\left[2^{1} 2^{0} \theta^{1}(1-\theta)^{0}\right]$ & 0.5 & 0.0 \\
\hline $5-10$ & $Z=\log _{10}\left[2^{0} 2^{1} \theta^{0}(1-\theta)^{1}\right]$ & 0.0 & 0.30 \\
\hline $6-7$ & $Z=\log _{10}\left[2^{0} 2^{1} \theta^{0}(1-\theta)^{1}\right]$ & 0.0 & 0.30 \\
\hline $6-8$ & $Z=\log _{10}\left[2^{0} 2^{1} \theta^{0}(1-\theta)^{1}\right]$ & 0.0 & 0.30 \\
\hline $6-9$ & $Z=\log _{10}\left[2^{0} 2^{1} \theta^{0}(1-\theta)^{1}\right]$ & 0.5 & 0.0 \\
\hline $6-10$ & $Z=\log _{10}\left[2^{0} 2^{1} \theta^{0}(1-\theta)^{1}\right]$ & 0.0 & 0.30 \\
\hline $7-8$ & $Z=\log _{10}\left[2^{0} 2^{1} \theta^{0}(1-\theta)^{1}\right]$ & 0.0 & 0.30 \\
\hline $7-9$ & $Z=\log _{10}\left[2^{1} 2^{0} \theta^{1}(1-\theta)^{0}\right]$ & 0.5 & 0.0 \\
\hline $7-10$ & $Z=\log _{10}\left[2^{0} 2^{1} \theta^{0}(1-\theta)^{1}\right]$ & 0.0 & 0.30 \\
\hline $8-9$ & $Z=\log _{10}\left[2^{1} 2^{0} \theta^{1}(1-\theta)^{0}\right]$ & 0.5 & 0.0 \\
\hline $8-10$ & $Z=\log _{10}\left[2^{0} 2^{1} \theta^{0}(1-\theta)^{1}\right]$ & 0.0 & 0.30 \\
\hline
\end{tabular}

Table 17. Linkage analysis of family 13.

\begin{tabular}{cccc}
\hline Combination of Markers & LOD & $\theta_{\text {max }}$ & $Z_{\text {max }}^{(13)}$ \\
\hline $1-2$ & $Z=\log _{10}\left[2^{1} 2^{0} \theta^{1}(1-\theta)^{0}\right]$ & 0.5 & 0.0 \\
$1-3$ & $Z=\log _{10}\left[2^{0} 2^{1} \theta^{0}(1-\theta)^{1}\right]$ & 0.0 & 0.30 \\
$1-4$ & $Z=\log _{10}\left[2^{0} 2^{1} \theta^{0}(1-\theta)^{1}\right]$ & 0.0 & 0.30 \\
$1-5$ & $Z=\log _{10}\left[2^{0} 2^{1} \theta^{0}(1-\theta)^{1}\right]$ & 0.0 & 0.30 \\
$1-6$ & $Z=\log _{10}\left[2^{0} 2^{1} \theta^{0}(1-\theta)^{1}\right]$ & 0.0 & 0.30 \\
$1-7$ & $Z=\log _{10}\left[2^{1} 2^{0} \theta^{1}(1-\theta)^{0}\right]$ & 0.5 & 0.0 \\
$1-8$ & $Z=\log _{10}\left[2^{0} 2^{1} \theta^{0}(1-\theta)^{1}\right]$ & 0.0 & 0.30 \\
$1-10$ & $Z=\log _{10}\left[2^{0} 2^{1} \theta^{0}(1-\theta)^{1}\right]$ & 0.0 & 0.30 \\
\hline
\end{tabular}




\section{Continued}

\begin{tabular}{|c|c|c|c|}
\hline $2-3$ & $Z=\log _{10}\left[2^{1} 2^{0} \theta^{1}(1-\theta)^{0}\right]$ & 0.5 & 0.0 \\
\hline $2-4$ & $Z=\log _{10}\left[2^{1} 2^{0} \theta^{1}(1-\theta)^{0}\right]$ & 0.5 & 0.0 \\
\hline $2-5$ & $Z=\log _{10}\left[2^{1} 2^{0} \theta^{1}(1-\theta)^{0}\right]$ & 0.5 & 0.0 \\
\hline $2-6$ & $Z=\log _{10}\left[2^{1} 2^{0} \theta^{1}(1-\theta)^{0}\right]$ & 0.5 & 0.0 \\
\hline $2-7$ & $Z=\log _{10}\left[2^{1} 2^{0} \theta^{1}(1-\theta)^{0}\right]$ & 0.5 & 0.0 \\
\hline $2-8$ & $Z=\log _{10}\left[2^{1} 2^{0} \theta^{1}(1-\theta)^{0}\right]$ & 0.5 & 0.0 \\
\hline $2-9$ & $Z=\log _{10}\left[2^{1} 2^{0} \theta^{1}(1-\theta)^{0}\right]$ & 0.5 & 0.0 \\
\hline $2-10$ & $Z=\log _{10}\left[2^{1} 2^{0} \theta^{1}(1-\theta)^{0}\right]$ & 0.5 & 0.0 \\
\hline $3-4$ & $Z=\log _{10}\left[2^{0} 2^{1} \theta^{0}(1-\theta)^{1}\right]$ & 0.0 & 0.30 \\
\hline $3-5$ & $Z=\log _{10}\left[2^{0} 2^{1} \theta^{0}(1-\theta)^{1}\right]$ & 0.0 & 0.30 \\
\hline $3-6$ & $Z=\log _{10}\left[2^{0} 2^{1} \theta^{0}(1-\theta)^{1}\right]$ & 0.0 & 0.30 \\
\hline $3-7$ & $Z=\log _{10}\left[2^{1} 2^{0} \theta^{1}(1-\theta)^{0}\right]$ & 0.5 & 0.0 \\
\hline $3-8$ & $Z=\log _{10}\left[2^{0} 2^{1} \theta^{0}(1-\theta)^{1}\right]$ & 0.0 & 0.30 \\
\hline $3-9$ & $Z=\log _{10}\left[2^{0} 2^{1} \theta^{0}(1-\theta)^{1}\right]$ & 0.0 & 0.30 \\
\hline $3-10$ & $Z=\log _{10}\left[2^{0} 2^{1} \theta^{0}(1-\theta)^{1}\right]$ & 0.0 & 0.30 \\
\hline $4-5$ & $Z=\log _{10}\left[2^{0} 2^{1} \theta^{0}(1-\theta)^{1}\right]$ & 0.0 & 0.30 \\
\hline $4-6$ & $Z=\log _{10}\left[2^{0} 2^{1} \theta^{0}(1-\theta)^{1}\right]$ & 0.0 & 0.30 \\
\hline $4-7$ & $Z=\log _{10}\left[2^{1} 2^{0} \theta^{1}(1-\theta)^{0}\right]$ & 0.5 & 0.0 \\
\hline $4-8$ & $Z=\log _{10}\left[2^{0} 2^{1} \theta^{0}(1-\theta)^{1}\right]$ & 0.0 & 0.30 \\
\hline $4-9$ & $Z=\log _{10}\left[2^{0} 2^{1} \theta^{0}(1-\theta)^{1}\right]$ & 0.0 & 0.30 \\
\hline $4-10$ & $Z=\log _{10}\left[2^{0} 2^{1} \theta^{0}(1-\theta)^{1}\right]$ & 0.0 & 0.30 \\
\hline $5-6$ & $Z=\log _{10}\left[2^{0} 2^{1} \theta^{0}(1-\theta)^{1}\right]$ & 0.0 & 0.30 \\
\hline $5-7$ & $Z=\log _{10}\left[2^{1} 2^{0} \theta^{1}(1-\theta)^{0}\right]$ & 0.5 & 0.0 \\
\hline $5-8$ & $Z=\log _{10}\left[2^{0} 2^{1} \theta^{0}(1-\theta)^{1}\right]$ & 0.0 & 0.30 \\
\hline $5-9$ & $Z=\log _{10}\left[2^{0} 2^{1} \theta^{0}(1-\theta)^{1}\right]$ & 0.0 & 0.30 \\
\hline $5-10$ & $Z=\log _{10}\left[2^{0} 2^{1} \theta^{0}(1-\theta)^{1}\right]$ & 0.0 & 0.30 \\
\hline $6-7$ & $Z=\log _{10}\left[2^{0} 2^{1} \theta^{0}(1-\theta)^{1}\right]$ & 0.5 & 0.0 \\
\hline $6-8$ & $Z=\log _{10}\left[2^{0} 2^{1} \theta^{0}(1-\theta)^{1}\right]$ & 0.0 & 0.30 \\
\hline $6-9$ & $Z=\log _{10}\left[2^{0} 2^{1} \theta^{0}(1-\theta)^{1}\right]$ & 0.0 & 0.30 \\
\hline $6-10$ & $Z=\log _{10}\left[2^{0} 2^{1} \theta^{0}(1-\theta)^{1}\right]$ & 0.0 & 0.30 \\
\hline $7-8$ & $Z=\log _{10}\left[2^{1} 2^{0} \theta^{1}(1-\theta)^{0}\right]$ & 0.5 & 0.0 \\
\hline
\end{tabular}




\section{Continued}

\begin{tabular}{cccc}
\hline $7-9$ & $Z=\log _{10}\left[2^{1} 2^{0} \theta^{1}(1-\theta)^{0}\right]$ & 0.5 & 0.0 \\
$7-10$ & $Z=\log _{10}\left[2^{1} 2^{0} \theta^{1}(1-\theta)^{0}\right]$ & 0.5 & 0.0 \\
$8-9$ & $Z=\log _{10}\left[2^{0} 2^{1} \theta^{0}(1-\theta)^{1}\right]$ & 0.0 & 0.30 \\
$8-10$ & $Z=\log _{10}\left[2^{0} 2^{1} \theta^{0}(1-\theta)^{1}\right]$ & 0.0 & 0.30 \\
\hline
\end{tabular}

Table 18. Linkage analysis of family 14.

\begin{tabular}{|c|c|c|c|}
\hline Combination of Markers & LOD & $\theta_{\max }$ & $Z_{\max }^{(14)}$ \\
\hline $1-2$ & $Z=\log _{10}\left[2^{1} 2^{0} \theta^{1}(1-\theta)^{0}\right]$ & 0.5 & 0.0 \\
\hline $1-3$ & $Z=\log _{10}\left[2^{1} 2^{0} \theta^{1}(1-\theta)^{0}\right]$ & 0.5 & 0.0 \\
\hline $1-4$ & $Z=\log _{10}\left[2^{1} 2^{0} \theta^{1}(1-\theta)^{0}\right]$ & 0.5 & 0.0 \\
\hline $1-5$ & $Z=\log _{10}\left[2^{1} 2^{0} \theta^{1}(1-\theta)^{0}\right]$ & 0.5 & 0.0 \\
\hline $1-6$ & $Z=\log _{10}\left[2^{1} 2^{0} \theta^{1}(1-\theta)^{0}\right]$ & 0.5 & 0.0 \\
\hline $1-7$ & $Z=\log _{10}\left[2^{1} 2^{0} \theta^{1}(1-\theta)^{0}\right]$ & 0.5 & 0.0 \\
\hline $1-8$ & $Z=\log _{10}\left[2^{1} 2^{0} \theta^{1}(1-\theta)^{0}\right]$ & 0.5 & 0.0 \\
\hline $1-9$ & $Z=\log _{10}\left[2^{1} 2^{0} \theta^{1}(1-\theta)^{0}\right]$ & 0.5 & 0.0 \\
\hline $1-10$ & $Z=\log _{10}\left[2^{1} 2^{0} \theta^{1}(1-\theta)^{0}\right]$ & 0.5 & 0.0 \\
\hline $2-3$ & $Z=\log _{10}\left[2^{1} 2^{0} \theta^{1}(1-\theta)^{0}\right]$ & 0.5 & 0.0 \\
\hline $2-4$ & $Z=\log _{10}\left[2^{1} 2^{0} \theta^{1}(1-\theta)^{0}\right]$ & 0.5 & 0.0 \\
\hline $2-5$ & $Z=\log _{10}\left[2^{1} 2^{0} \theta^{1}(1-\theta)^{0}\right]$ & 0.5 & 0.0 \\
\hline $2-6$ & $Z=\log _{10}\left[2^{1} 2^{0} \theta^{1}(1-\theta)^{0}\right]$ & 0.5 & 0.0 \\
\hline $2-7$ & $Z=\log _{10}\left[2^{1} 2^{0} \theta^{1}(1-\theta)^{0}\right]$ & 0.5 & 0.0 \\
\hline $2-8$ & $Z=\log _{10}\left[2^{1} 2^{0} \theta^{1}(1-\theta)^{0}\right]$ & 0.5 & 0.0 \\
\hline $2-9$ & $Z=\log _{10}\left[2^{1} 2^{0} \theta^{1}(1-\theta)^{0}\right]$ & 0.5 & 0.0 \\
\hline $2-10$ & $Z=\log \left[2^{1} 2^{0} \theta^{1}(1-\theta)^{0}\right]$ & 0.5 & 0.0 \\
\hline $3-4$ & $Z=\log _{10}\left[2^{0} 2^{1} \theta^{0}(1-\theta)^{1}\right]$ & 0.0 & 0.30 \\
\hline $3-5$ & $Z=\log _{10}\left[2^{1} 2^{0} \theta^{1}(1-\theta)^{0}\right]$ & 0.5 & 0.0 \\
\hline $3-6$ & $Z=\log _{10}\left[2^{0} 2^{1} \theta^{0}(1-\theta)^{1}\right]$ & 0.0 & 0.30 \\
\hline $3-7$ & $Z=\log \left[2^{0} 2^{1} \theta^{0}(1-\theta)^{1}\right]$ & 0.0 & 0.30 \\
\hline $3-8$ & $Z=\log _{0}\left[2^{0} 2^{1} \theta^{0}(1-\theta)^{1}\right]$ & 0.0 & 0.30 \\
\hline $3-9$ & $Z=\log \left[2^{0} 2^{1} \theta^{0}(1-\theta)^{1}\right]$ & 0.0 & 0.30 \\
\hline $3-10$ & $Z=\log _{10}\left[2^{0} 2^{1} \theta^{0}(1-\theta)^{1}\right]$ & 0.0 & 0.30 \\
\hline
\end{tabular}




\section{Continued}

\begin{tabular}{|c|c|c|c|}
\hline $4-5$ & $Z=\log _{10}\left[2^{1} 2^{0} \theta^{1}(1-\theta)^{0}\right]$ & 0.5 & 0.0 \\
\hline $4-6$ & $Z=\log _{10}\left[2^{0} 2^{1} \theta^{0}(1-\theta)^{1}\right]$ & 0.0 & 0.30 \\
\hline $4-7$ & $Z=\log _{10}\left[2^{0} 2^{1} \theta^{0}(1-\theta)^{1}\right]$ & 0.0 & 0.30 \\
\hline $4-8$ & $Z=\log _{10}\left[2^{0} 2^{1} \theta^{0}(1-\theta)^{1}\right]$ & 0.0 & 0.30 \\
\hline $4-9$ & $Z=\log _{10}\left[2^{0} 2^{1} \theta^{0}(1-\theta)^{1}\right]$ & 0.0 & 0.30 \\
\hline $4-10$ & $Z=\log _{10}\left[2^{0} 2^{1} \theta^{0}(1-\theta)^{1}\right]$ & 0.0 & 0.30 \\
\hline $5-6$ & $Z=\log _{10}\left[2^{1} 2^{0} \theta^{1}(1-\theta)^{0}\right]$ & 0.5 & 0.0 \\
\hline $5-7$ & $Z=\log _{10}\left[2^{1} 2^{0} \theta^{1}(1-\theta)^{0}\right]$ & 0.5 & 0.0 \\
\hline $5-8$ & $Z=\log _{10}\left[2^{1} 2^{0} \theta^{1}(1-\theta)^{0}\right]$ & 0.5 & 0.0 \\
\hline $5-9$ & $Z=\log _{10}\left[2^{1} 2^{0} \theta^{1}(1-\theta)^{0}\right]$ & 0.5 & 0.0 \\
\hline $5-10$ & $Z=\log _{10}\left[2^{1} 2^{0} \theta^{1}(1-\theta)^{0}\right]$ & 0.5 & 0.0 \\
\hline $6-7$ & $Z=\log _{10}\left[2^{0} 2^{1} \theta^{0}(1-\theta)^{1}\right.$ & 0.0 & 0.30 \\
\hline $6-8$ & $Z=\log _{10}\left[2^{0} 2^{1} \theta^{0}(1-\theta)^{1}\right]$ & 0.0 & 0.30 \\
\hline $6-9$ & $Z=\log _{10}\left[2^{0} 2^{1} \theta^{0}(1-\theta)^{1}\right]$ & 0.0 & 0.30 \\
\hline $6-10$ & $Z=\log _{10}\left[2^{0} 2^{1} \theta^{0}(1-\theta)^{1}\right]$ & 0.0 & 0.30 \\
\hline $7-8$ & $Z=\log _{10}\left[2^{0} 2^{1} \theta^{0}(1-\theta)^{1}\right]$ & 0.0 & 0.30 \\
\hline $7-9$ & $Z=\log _{10}\left[2^{0} 2^{1} \theta^{0}(1-\theta)^{1}\right]$ & 0.0 & 0.30 \\
\hline $7-10$ & $Z=\log _{10}\left[2^{0} 2^{1} \theta^{0}(1-\theta)^{1}\right]$ & 0.0 & 0.30 \\
\hline $8-9$ & $Z=\log _{10}\left[2^{0} 2^{1} \theta^{0}(1-\theta)^{1}\right]$ & 0.0 & 0.30 \\
\hline $8-10$ & $Z=\log _{10}\left[2^{0} 2^{1} \theta^{0}(1-\theta)^{1}\right.$ & 0.0 & 0.30 \\
\hline
\end{tabular}

Table 19. Linkage analysis of family 15.

\begin{tabular}{cccc}
\hline Combination of Markers & LOD & $\theta_{\max }$ & $Z_{\text {max }}^{(15)}$ \\
\hline $1-2$ & $Z=\log _{10}\left[2^{0} 2^{1} \theta^{0}(1-\theta)^{1}\right]$ & 0.0 & 0.30 \\
$1-3$ & $Z=\log _{10}\left[2^{0} 2^{1} \theta^{0}(1-\theta)^{1}\right]$ & 0.0 & 0.30 \\
$1-4$ & $Z=\log _{10}\left[2^{1} 2^{0} \theta^{1}(1-\theta)^{0}\right]$ & 0.5 & 0.0 \\
$1-5$ & $Z=\log _{10}\left[2^{1} 2^{0} \theta^{1}(1-\theta)^{0}\right]$ & 0.5 & 0.0 \\
$1-6$ & $Z=\log _{10}\left[2^{1} 2^{0} \theta^{1}(1-\theta)^{0}\right]$ & 0.5 & 0.0 \\
$1-7$ & $Z=\log _{10}\left[2^{1} 2^{0} \theta^{1}(1-\theta)^{0}\right]$ & 0.5 & 0.0 \\
$1-8$ & $Z=\log _{10}\left[2^{0} 2^{1} \theta^{0}(1-\theta)^{1}\right]$ & 0.0 & 0.30 \\
$1-10$ & $Z=\log _{10}\left[2^{0} 2^{1} \theta^{0}(1-\theta)^{1}\right]$ & 0.0 & 0.30 \\
\hline
\end{tabular}




\section{Continued}

\begin{tabular}{|c|c|c|c|}
\hline $2-3$ & $Z=\log _{10}\left[2^{0} 2^{1} \theta^{0}(1-\theta)^{1}\right]$ & 0.0 & 0.30 \\
\hline $2-4$ & $Z=\log _{10}\left[2^{1} 2^{0} \theta^{1}(1-\theta)^{0}\right]$ & 0.5 & 0.0 \\
\hline $2-5$ & $Z=\log _{10}\left[2^{1} 2^{0} \theta^{1}(1-\theta)^{0}\right]$ & 0.5 & 0.0 \\
\hline $2-6$ & $Z=\log _{10}\left[2^{1} 2^{0} \theta^{1}(1-\theta)^{0}\right]$ & 0.5 & 0.0 \\
\hline $2-7$ & $Z=\log _{10}\left[2^{1} 2^{0} \theta^{1}(1-\theta)^{0}\right]$ & 0.5 & 0.0 \\
\hline $2-8$ & $Z=\log _{10}\left[2^{0} 2^{1} \theta^{0}(1-\theta)^{1}\right]$ & 0.0 & 0.30 \\
\hline $2-9$ & $Z=\log _{10}\left[2^{0} 2^{1} \theta^{0}(1-\theta)^{1}\right]$ & 0.0 & 0.30 \\
\hline $2-10$ & $Z=\log _{10}\left[2^{0} 2^{1} \theta^{0}(1-\theta)^{1}\right]$ & 0.0 & 0.30 \\
\hline $3-4$ & $Z=\log _{10}\left[2^{1} 2^{0} \theta^{1}(1-\theta)^{0}\right]$ & 0.5 & 0.0 \\
\hline $3-5$ & $Z=\log _{10}\left[2^{1} 2^{0} \theta^{1}(1-\theta)^{0}\right]$ & 0.5 & 0.0 \\
\hline $3-6$ & $Z=\log _{10}\left[2^{1} 2^{0} \theta^{1}(1-\theta)^{0}\right]$ & 0.5 & 0.0 \\
\hline $3-7$ & $Z=\log _{10}\left[2^{1} 2^{0} \theta^{1}(1-\theta)^{0}\right]$ & 0.5 & 0.0 \\
\hline $3-8$ & $Z=\log _{10}\left[2^{0} 2^{1} \theta^{0}(1-\theta)^{1}\right.$ & 0.0 & 0.30 \\
\hline $3-9$ & $Z=\log _{10}\left[2^{0} 2^{1} \theta^{0}(1-\theta)^{1}\right.$ & 0.0 & 0.30 \\
\hline $3-10$ & $Z=\log _{10}\left[2^{0} 2^{1} \theta^{0}(1-\theta)^{1}\right]$ & 0.0 & 0.30 \\
\hline $4-5$ & $Z=\log _{10}\left[2^{1} 2^{0} \theta^{1}(1-\theta)^{0}\right]$ & 0.5 & 0.0 \\
\hline $4-6$ & $Z=\log _{10}\left[2^{1} 2^{0} \theta^{1}(1-\theta)^{0}\right.$ & 0.5 & 0.0 \\
\hline $4-7$ & $Z=\log _{10}\left[2^{1} 2^{0} \theta^{1}(1-\theta)^{0}\right]$ & 0.5 & 0.0 \\
\hline $4-8$ & $Z=\log _{10}\left[2^{1} 2^{0} \theta^{1}(1-\theta)^{0}\right]$ & 0.5 & 0.0 \\
\hline $4-9$ & $Z=\log _{10}\left[2^{1} 2^{0} \theta^{1}(1-\theta)^{0}\right.$ & 0.5 & 0.0 \\
\hline $4-10$ & $Z=\log _{10}\left[2^{1} 2^{0} \theta^{1}(1-\theta)^{0}\right]$ & 0.5 & 0.0 \\
\hline $5-6$ & $Z=\log _{10}\left[2^{0} 2^{1} \theta^{0}(1-\theta)^{1}\right.$ & 0.0 & 0.30 \\
\hline $5-7$ & $Z=\log _{10}\left[2^{0} 2^{1} \theta^{0}(1-\theta)^{1}\right]$ & 0.0 & 0.30 \\
\hline $5-8$ & $Z=\log _{10}\left[2^{0} 2^{1} \theta^{0}(1-\theta)^{1}\right]$ & 0.0 & 0.30 \\
\hline $5-9$ & $Z=\log _{10}\left[2^{0} 2^{1} \theta^{0}(1-\theta)^{1}\right]$ & 0.0 & 0.30 \\
\hline $5-10$ & $Z=\log _{10}\left[2^{0} 2^{1} \theta^{0}(1-\theta)^{1}\right]$ & 0.0 & 0.30 \\
\hline $6-7$ & $Z=\log _{10}\left[2^{1} 2^{0} \theta^{1}(1-\theta)^{0}\right]$ & 0.5 & 0.0 \\
\hline $6-8$ & $Z=\log _{10}\left[2^{1} 2^{0} \theta^{1}(1-\theta)^{0}\right]$ & 0.5 & 0.0 \\
\hline $6-9$ & $Z=\log _{10}\left[2^{1} 2^{0} \theta^{1}(1-\theta)^{0}\right.$ & 0.5 & 0.0 \\
\hline $6-10$ & $Z=\log _{10}\left[2^{1} 2^{0} \theta^{1}(1-\theta)^{0}\right.$ & 0.5 & 0.0 \\
\hline $7-8$ & $Z=\log _{10}\left[2^{1} 2^{0} \theta^{1}(1-\theta)^{0}\right]$ & 0.5 & 0.0 \\
\hline
\end{tabular}




\section{Continued}

\begin{tabular}{|c|c|c|c|}
\hline $7-9$ & $Z=\log _{10}\left[2^{1} 2^{0} \theta^{1}(1-\theta)^{0}\right]$ & 0.5 & 0.0 \\
\hline $7-10$ & $Z=\log _{10}\left[2^{1} 2^{0} \theta^{1}(1-\theta)^{0}\right]$ & 0.5 & 0.0 \\
\hline $8-9$ & $Z=\log _{10}\left[2^{0} 2^{1} \theta^{0}(1-\theta)^{1}\right]$ & 0.0 & 0.30 \\
\hline $8-10$ & $Z=\log _{10}\left[2^{0} 2^{1} \theta^{0}(1-\theta)^{1}\right]$ & 0.0 & 0.30 \\
\hline
\end{tabular}

Table 20. Linkage analysis of family 16.

\begin{tabular}{|c|c|c|c|}
\hline Combination of Markers & LOD & $\theta_{\max }$ & $Z_{\max }^{(16)}$ \\
\hline $1-2$ & $Z=\log _{10}\left[2^{1} 2^{0} \theta^{1}(1-\theta)^{0}\right]$ & 0.5 & 0.0 \\
\hline $1-3$ & $Z=\log _{10}\left[2^{1} 2^{0} \theta^{1}(1-\theta)^{0}\right]$ & 0.5 & 0.0 \\
\hline $1-4$ & $Z=\log _{10}\left[2^{1} 2^{0} \theta^{1}(1-\theta)^{0}\right]$ & 0.5 & 0.0 \\
\hline $1-5$ & $Z=\log _{10}\left[2^{1} 2^{0} \theta^{1}(1-\theta)^{0}\right]$ & 0.5 & 0.0 \\
\hline $1-6$ & $Z=\log _{10}\left[2^{1} 2^{0} \theta^{1}(1-\theta)^{0}\right]$ & 0.5 & 0.0 \\
\hline $1-7$ & $Z=\log _{10}\left[2^{1} 2^{0} \theta^{1}(1-\theta)^{0}\right]$ & 0.5 & 0.0 \\
\hline $1-8$ & $Z=\log _{10}\left[2^{1} 2^{0} \theta^{1}(1-\theta)^{0}\right]$ & 0.5 & 0.0 \\
\hline $1-9$ & $Z=\log _{10}\left[2^{1} 2^{0} \theta^{1}(1-\theta)^{0}\right]$ & 0.5 & 0.0 \\
\hline $1-10$ & $Z=\log _{10}\left[2^{1} 2^{0} \theta^{1}(1-\theta)^{0}\right]$ & 0.5 & 0.0 \\
\hline $2-3$ & $Z=\log _{10}\left[2^{1} 2^{0} \theta^{1}(1-\theta)^{0}\right]$ & 0.5 & 0.0 \\
\hline $2-4$ & $Z=\log _{10}\left[2^{1} 2^{0} \theta^{1}(1-\theta)^{0}\right]$ & 0.5 & 0.0 \\
\hline $2-5$ & $Z=\log _{10}\left[2^{1} 2^{0} \theta^{1}(1-\theta)^{0}\right]$ & 0.5 & 0.0 \\
\hline $2-6$ & $Z=\log _{10}\left[2^{1} 2^{0} \theta^{1}(1-\theta)^{0}\right]$ & 0.5 & 0.0 \\
\hline $2-7$ & $Z=\log _{10}\left[2^{1} 2^{0} \theta^{1}(1-\theta)^{0}\right]$ & 0.5 & 0.0 \\
\hline $2-8$ & $Z=\log _{10}\left[2^{1} 2^{0} \theta^{1}(1-\theta)^{0}\right]$ & 0.5 & 0.0 \\
\hline $2-9$ & $Z=\log _{10}\left[2^{1} 2^{0} \theta^{1}(1-\theta)^{0}\right]$ & 0.5 & 0.0 \\
\hline $2-10$ & $Z=\log _{10}\left[2^{1} 2^{0} \theta^{1}(1-\theta)^{0}\right]$ & 0.5 & 0.0 \\
\hline $3-4$ & $Z=\log _{10}\left[2^{0} 2^{1} \theta^{0}(1-\theta)^{1}\right]$ & 0.0 & 0.30 \\
\hline $3-5$ & $Z=\log _{10}\left[2^{1} 2^{0} \theta^{1}(1-\theta)^{0}\right]$ & 0.5 & 0.0 \\
\hline $3-6$ & $Z=\log _{10}\left[2^{0} 2^{1} \theta^{0}(1-\theta)^{1}\right]$ & 0.0 & 0.30 \\
\hline $3-7$ & $Z=\log _{10}\left[2^{1} 2^{0} \theta^{1}(1-\theta)^{0}\right]$ & 0.5 & 0.0 \\
\hline $3-8$ & $Z=\log _{10}\left[2^{0} 2^{1} \theta^{0}(1-\theta)^{1}\right]$ & 0.0 & 0.30 \\
\hline $3-9$ & $Z=\log _{10}\left[2^{0} 2^{1} \theta^{0}(1-\theta)^{1}\right]$ & 0.0 & 0.30 \\
\hline $3-10$ & $Z=\log _{10}\left[2^{1} 2^{0} \theta^{1}(1-\theta)^{0}\right]$ & 0.5 & 0.0 \\
\hline
\end{tabular}




\section{Continued}

\begin{tabular}{|c|c|c|c|}
\hline $4-5$ & $Z=\log _{10}\left[2^{1} 2^{0} \theta^{1}(1-\theta)^{0}\right]$ & 0.5 & 0.0 \\
\hline $4-6$ & $Z=\log _{10}\left[2^{0} 2^{1} \theta^{0}(1-\theta)^{1}\right]$ & 0.0 & 0.30 \\
\hline $4-7$ & $Z=\log _{10}\left[2^{1} 2^{0} \theta^{1}(1-\theta)^{0}\right]$ & 0.5 & 0.0 \\
\hline $4-8$ & $Z=\log _{10}\left[2^{0} 2^{1} \theta^{0}(1-\theta)^{1}\right]$ & 0.0 & 0.30 \\
\hline $4-9$ & $Z=\log _{10}\left[2^{0} 2^{1} \theta^{0}(1-\theta)^{1}\right]$ & 0.0 & 0.30 \\
\hline $4-10$ & $Z=\log _{10}\left[2^{1} 2^{0} \theta^{1}(1-\theta)^{0}\right]$ & 0.5 & 0.0 \\
\hline $5-6$ & $Z=\log _{10}\left[2^{1} 2^{0} \theta^{1}(1-\theta)^{0}\right]$ & 0.5 & 0.0 \\
\hline $5-7$ & $Z=\log _{10}\left[2^{1} 2^{0} \theta^{1}(1-\theta)^{0}\right]$ & 0.5 & 0.0 \\
\hline $5-8$ & $Z=\log _{10}\left[2^{1} 2^{0} \theta^{1}(1-\theta)^{0}\right]$ & 0.5 & 0.0 \\
\hline $5-9$ & $Z=\log _{10}\left[2^{1} 2^{0} \theta^{1}(1-\theta)^{0}\right]$ & 0.5 & 0.0 \\
\hline $5-10$ & $Z=\log _{10}\left[2^{1} 2^{0} \theta^{1}(1-\theta)^{0}\right]$ & 0.5 & 0.0 \\
\hline $6-7$ & $Z=\log _{10}\left[2^{1} 2^{0} \theta^{1}(1-\theta)^{0}\right]$ & 0.5 & 0.0 \\
\hline $6-8$ & $Z=\log _{10}\left[2^{0} 2^{1} \theta^{0}(1-\theta)^{1}\right]$ & 0.0 & 0.30 \\
\hline $6-9$ & $Z=\log _{10}\left[2^{0} 2^{1} \theta^{0}(1-\theta)^{1}\right]$ & 0.0 & 0.30 \\
\hline $6-10$ & $Z=\log _{10}\left[2^{1} 2^{0} \theta^{1}(1-\theta)^{0}\right]$ & 0.5 & 0.0 \\
\hline $7-8$ & $Z=\log _{10}\left[2^{1} 2^{0} \theta^{1}(1-\theta)^{0}\right]$ & 0.5 & 0.0 \\
\hline $7-9$ & $Z=\log _{10}\left[2^{1} 2^{0} \theta^{1}(1-\theta)^{0}\right]$ & 0.5 & 0.0 \\
\hline $7-10$ & $Z=\log _{10}\left[2^{1} 2^{0} \theta^{1}(1-\theta)^{0}\right]$ & 0.5 & 0.0 \\
\hline $8-9$ & $Z=\log _{10}\left[2^{0} 2^{0} \theta^{0}(1-\theta)^{1}\right]$ & 0.0 & 0.30 \\
\hline $8-10$ & $Z=\log _{10}\left[2^{1} 2^{0} \theta^{1}(1-\theta)^{0}\right]$ & 0.5 & 0.0 \\
\hline
\end{tabular}

Table 21. Linkage analysis of family 17.

\begin{tabular}{cccc}
\hline Combination of Markers & LOD & $\theta_{\max }$ & $Z_{\text {max }}^{(17)}$ \\
\hline $1-2$ & $Z=\log _{10}\left[2^{0} 2^{1} \theta^{0}(1-\theta)^{1}\right]$ & 0.0 & 0.30 \\
$1-3$ & $Z=\log _{10}\left[2^{0} 2^{1} \theta^{0}(1-\theta)^{1}\right]$ & 0.0 & 0.30 \\
$1-4$ & $Z=\log _{10}\left[2^{0} 2^{1} \theta^{0}(1-\theta)^{1}\right]$ & 0.0 & 0.30 \\
$1-5$ & $Z=\log _{10}\left[2^{0} 2^{1} \theta^{0}(1-\theta)^{1}\right]$ & 0.0 & 0.30 \\
$1-6$ & $Z=\log _{10}\left[2^{0} 2^{1} \theta^{0}(1-\theta)^{1}\right]$ & 0.0 & 0.30 \\
$1-7$ & $Z=\log _{10}\left[2^{0} 2^{1} \theta^{0}(1-\theta)^{1}\right]$ & 0.0 & 0.30 \\
$1-8$ & $Z=\log _{10}\left[2^{0} 2^{1} \theta^{0}(1-\theta)^{1}\right]$ & 0.0 & 0.30 \\
\hline
\end{tabular}




\section{Continued}

\begin{tabular}{|c|c|c|c|}
\hline $1-10$ & $Z=\log _{10}\left[2^{0} 2^{1} \theta^{0}(1-\theta)^{1}\right]$ & 0.0 & 0.30 \\
\hline $2-3$ & $Z=\log _{10}\left[2^{0} 2^{1} \theta^{0}(1-\theta)^{1}\right]$ & 0.0 & 0.30 \\
\hline $2-4$ & $Z=\log _{10}\left[2^{0} 2^{1} \theta^{0}(1-\theta)^{1}\right]$ & 0.0 & 0.30 \\
\hline $2-5$ & $Z=\log _{10}\left[2^{0} 2^{1} \theta^{0}(1-\theta)^{1}\right]$ & 0.0 & 0.30 \\
\hline $2-6$ & $Z=\log _{10}\left[2^{0} 2^{1} \theta^{0}(1-\theta)^{1}\right]$ & 0.0 & 0.30 \\
\hline $2-7$ & $Z=\log _{10}\left[2^{0} 2^{1} \theta^{0}(1-\theta)^{1}\right]$ & 0.0 & 0.30 \\
\hline $2-8$ & $Z=\log _{10}\left[2^{0} 2^{1} \theta^{0}(1-\theta)^{1}\right]$ & 0.0 & 0.30 \\
\hline $2-9$ & $Z=\log _{10}\left[2^{0} 2^{1} \theta^{0}(1-\theta)^{1}\right]$ & 0.0 & 0.30 \\
\hline $2-10$ & $Z=\log _{10}\left[2^{0} 2^{1} \theta^{0}(1-\theta)^{1}\right]$ & 0.0 & 0.30 \\
\hline $3-4$ & $Z=\log _{10}\left[2^{0} 2^{1} \theta^{0}(1-\theta)^{1}\right]$ & 0.0 & 0.30 \\
\hline $3-5$ & $Z=\log _{10}\left[2^{0} 2^{1} \theta^{0}(1-\theta)^{1}\right]$ & 0.0 & 0.30 \\
\hline $3-6$ & $Z=\log _{10}\left[2^{0} 2^{1} \theta^{0}(1-\theta)^{1}\right]$ & 0.0 & 0.30 \\
\hline $3-7$ & $Z=\log _{10}\left[2^{0} 2^{1} \theta^{0}(1-\theta)^{1}\right]$ & 0.0 & 0.30 \\
\hline $3-8$ & $Z=\log _{10}\left[2^{0} 2^{1} \theta^{0}(1-\theta)^{1}\right]$ & 0.0 & 0.30 \\
\hline $3-9$ & $Z=\log _{10}\left[2^{0} 2^{1} \theta^{0}(1-\theta)^{1}\right]$ & 0.0 & 0.30 \\
\hline $3-10$ & $Z=\log _{10}\left[2^{0} 2^{1} \theta^{0}(1-\theta)^{1}\right]$ & 0.0 & 0.30 \\
\hline $4-5$ & $Z=\log _{10}\left[2^{0} 2^{1} \theta^{0}(1-\theta)^{1}\right]$ & 0.0 & 0.30 \\
\hline $4-6$ & $Z=\log _{10}\left[2^{0} 2^{1} \theta^{0}(1-\theta)^{1}\right]$ & 0.0 & 0.30 \\
\hline $4-7$ & $Z=\log _{10}\left[2^{0} 2^{1} \theta^{0}(1-\theta)^{1}\right]$ & 0.0 & 0.30 \\
\hline $4-8$ & $Z=\log _{10}\left[2^{0} 2^{1} \theta^{0}(1-\theta)^{1}\right]$ & 0.0 & 0.30 \\
\hline $4-9$ & $Z=\log _{10}\left[2^{0} 2^{1} \theta^{0}(1-\theta)^{1}\right]$ & 0.0 & 0.30 \\
\hline $4-10$ & $Z=\log _{10}\left[2^{0} 2^{1} \theta^{0}(1-\theta)^{1}\right]$ & 0.0 & 0.30 \\
\hline $5-6$ & $Z=\log _{10}\left[2^{0} 2^{1} \theta^{0}(1-\theta)^{1}\right]$ & 0.0 & 0.30 \\
\hline $5-7$ & $Z=\log _{10}\left[2^{0} 2^{1} \theta^{0}(1-\theta)^{1}\right]$ & 0.0 & 0.30 \\
\hline $5-8$ & $Z=\log _{10}\left[2^{0} 2^{1} \theta^{0}(1-\theta)^{1}\right]$ & 0.0 & 0.30 \\
\hline $5-9$ & $Z=\log _{10}\left[2^{0} 2^{1} \theta^{0}(1-\theta)^{1}\right]$ & 0.0 & 0.30 \\
\hline $5-10$ & $Z=\log _{10}\left[2^{0} 2^{1} \theta^{0}(1-\theta)^{1}\right]$ & 0.0 & 0.30 \\
\hline $6-7$ & $Z=\log _{10}\left[2^{0} 2^{1} \theta^{0}(1-\theta)^{1}\right]$ & 0.0 & 0.30 \\
\hline $6-8$ & $Z=\log _{10}\left[2^{0} 2^{1} \theta^{0}(1-\theta)^{1}\right]$ & 0.0 & 0.30 \\
\hline $6-9$ & $Z=\log _{10}\left[2^{0} 2^{1} \theta^{0}(1-\theta)^{1}\right]$ & 0.0 & 0.30 \\
\hline $6-10$ & $Z=\log _{10}\left[2^{0} 2^{1} \theta^{0}(1-\theta)^{1}\right]$ & 0.0 & 0.30 \\
\hline
\end{tabular}




\section{Continued}

$\begin{array}{llll}7-8 & Z=\log _{10}\left[2^{0} 2^{1} \theta^{0}(1-\theta)^{1}\right] & 0.0 & 0.30 \\ 7-9 & Z=\log _{10}\left[2^{0} 2^{1} \theta^{0}(1-\theta)^{1}\right] & 0.0 & 0.30 \\ 7-10 & Z=\log _{10}\left[2^{0} 2^{1} \theta^{0}(1-\theta)^{1}\right] & 0.0 & 0.30 \\ 8-9 & Z=\log _{10}\left[2^{0} 2^{1} \theta^{0}(1-\theta)^{1}\right] & 0.0 & 0.30 \\ 8-10 & Z=\log _{10}\left[2^{0} 2^{1} \theta^{0}(1-\theta)^{1}\right] & 0.0 & 0.30\end{array}$

Table 22. Linkage analysis of family 18.

\begin{tabular}{|c|c|c|c|}
\hline Combination of Markers & LOD & $\theta_{\max }$ & $Z_{\max }^{(18)}$ \\
\hline $1-2$ & $Z=\log _{10}\left[2^{1} 2^{0} \theta^{1}(1-\theta)^{0}\right]$ & 0.5 & 0.0 \\
\hline $1-3$ & $Z=\log _{10}\left[2^{1} 2^{0} \theta^{1}(1-\theta)^{0}\right]$ & 0.5 & 0.0 \\
\hline $1-4$ & $Z=\log _{10}\left[2^{1} 2^{0} \theta^{1}(1-\theta)^{0}\right]$ & 0.5 & 0.0 \\
\hline $1-5$ & $Z=\log _{10}\left[2^{1} 2^{0} \theta^{1}(1-\theta)^{0}\right]$ & 0.5 & 0.0 \\
\hline $1-6$ & $Z=\log _{10}\left[2^{1} 2^{0} \theta^{1}(1-\theta)^{0}\right]$ & 0.5 & 0.0 \\
\hline $1-7$ & $Z=\log _{10}\left[2^{1} 2^{0} \theta^{1}(1-\theta)^{0}\right]$ & 0.5 & 0.0 \\
\hline $1-8$ & $Z=\log _{10}\left[2^{1} 2^{0} \theta^{1}(1-\theta)^{0}\right]$ & 0.5 & 0.0 \\
\hline $1-9$ & $Z=\log _{10}\left[2^{1} 2^{0} \theta^{1}(1-\theta)^{0}\right]$ & 0.5 & 0.0 \\
\hline $1-10$ & $Z=\log _{10}\left[2^{1} 2^{0} \theta^{1}(1-\theta)^{0}\right]$ & 0.5 & 0.0 \\
\hline $2-3$ & $Z=\log _{10}\left[2^{1} 2^{0} \theta^{1}(1-\theta)^{0}\right]$ & 0.5 & 0.0 \\
\hline $2-4$ & $Z=\log _{10}\left[2^{1} 2^{0} \theta^{1}(1-\theta)^{0}\right]$ & 0.5 & 0.0 \\
\hline $2-5$ & $Z=\log _{10}\left[2^{1} 2^{0} \theta^{1}(1-\theta)^{0}\right]$ & 0.5 & 0.0 \\
\hline $2-6$ & $Z=\log _{10}\left[2^{0} 2^{1} \theta^{0}(1-\theta)^{1}\right]$ & 0.0 & 0.30 \\
\hline $2-7$ & $Z=\log _{10}\left[2^{1} 2^{0} \theta^{1}(1-\theta)^{0}\right]$ & 0.5 & 0.0 \\
\hline $2-8$ & $Z=\log _{10}\left[2^{1} 2^{0} \theta^{1}(1-\theta)^{0}\right]$ & 0.5 & 0.0 \\
\hline $2-9$ & $Z=\log _{10}\left[2^{0} 2^{1} \theta^{0}(1-\theta)^{1}\right]$ & 0.0 & 0.30 \\
\hline $2-10$ & $Z=\log _{10}\left[2^{0} 2^{1} \theta^{0}(1-\theta)^{1}\right]$ & 0.0 & 0.30 \\
\hline $3-4$ & $Z=\log _{10}\left[2^{1} 2^{0} \theta^{1}(1-\theta)^{0}\right]$ & 0.5 & 0.0 \\
\hline $3-5$ & $Z=\log _{10}\left[2^{1} 2^{0} \theta^{1}(1-\theta)^{0}\right]$ & 0.5 & 0.0 \\
\hline $3-6$ & $Z=\log _{10}\left[2^{1} 2^{0} \theta^{1}(1-\theta)^{0}\right]$ & 0.5 & 0.0 \\
\hline $3-7$ & $Z=\log _{10}\left[2^{1} 2^{0} \theta^{1}(1-\theta)^{0}\right]$ & 0.5 & 0.0 \\
\hline $3-8$ & $Z=\log _{10}\left[2^{1} 2^{0} \theta^{1}(1-\theta)^{0}\right]$ & 0.5 & 0.0 \\
\hline $3-9$ & $Z=\log _{10}\left[2^{1} 2^{0} \theta^{1}(1-\theta)^{0}\right]$ & 0.5 & 0.0 \\
\hline
\end{tabular}




\section{Continued}

\begin{tabular}{|c|c|c|c|}
\hline $3-10$ & $Z=\log _{10}\left[2^{1} 2^{0} \theta^{1}(1-\theta)^{0}\right]$ & 0.5 & 0.0 \\
\hline $4-5$ & $Z=\log _{10}\left[2^{1} 2^{0} \theta^{1}(1-\theta)^{0}\right]$ & 0.5 & 0.0 \\
\hline $4-6$ & $Z=\log _{10}\left[2^{1} 2^{0} \theta^{1}(1-\theta)^{0}\right]$ & 0.5 & 0.0 \\
\hline $4-7$ & $Z=\log _{10}\left[2^{1} 2^{0} \theta^{1}(1-\theta)^{0}\right]$ & 0.5 & 0.0 \\
\hline $4-8$ & $Z=\log _{10}\left[2^{1} 2^{0} \theta^{1}(1-\theta)^{0}\right]$ & 0.5 & 0.0 \\
\hline $4-9$ & $Z=\log _{10}\left[2^{1} 2^{0} \theta^{1}(1-\theta)^{0}\right]$ & 0.5 & 0.0 \\
\hline $4-10$ & $Z=\log _{10}\left[2^{1} 2^{0} \theta^{1}(1-\theta)^{0}\right]$ & 0.5 & 0.0 \\
\hline $5-6$ & $Z=\log _{10}\left[2^{1} 2^{0} \theta^{1}(1-\theta)^{0}\right]$ & 0.5 & 0.0 \\
\hline $5-7$ & $Z=\log _{10}\left[2^{1} 2^{0} \theta^{1}(1-\theta)^{0}\right]$ & 0.5 & 0.0 \\
\hline $5-8$ & $Z=\log _{10}\left[2^{1} 2^{0} \theta^{1}(1-\theta)^{0}\right]$ & 0.5 & 0.0 \\
\hline $5-9$ & $Z=\log _{10}\left[2^{1} 2^{0} \theta^{1}(1-\theta)^{0}\right]$ & 0.5 & 0.0 \\
\hline $5-10$ & $Z=\log _{10}\left[2^{1} 2^{0} \theta^{1}(1-\theta)^{0}\right]$ & 0.5 & 0.0 \\
\hline $6-7$ & $Z=\log _{10}\left[2^{1} 2^{0} \theta^{1}(1-\theta)^{0}\right]$ & 0.5 & 0.0 \\
\hline $6-8$ & $Z=\log _{10}\left[2^{1} 2^{0} \theta^{1}(1-\theta)^{0}\right]$ & 0.5 & 0.0 \\
\hline $6-9$ & $Z=\log _{10}\left[2^{0} 2^{1} \theta^{0}(1-\theta)^{1}\right]$ & 0.0 & 0.30 \\
\hline $6-10$ & $Z=\log _{10}\left[2^{0} 2^{1} \theta^{0}(1-\theta)^{1}\right]$ & 0.0 & 0.30 \\
\hline $7-8$ & $Z=\log _{10}\left[2^{1} 2^{0} \theta^{1}(1-\theta)^{0}\right]$ & 0.5 & 0.0 \\
\hline $7-9$ & $Z=\log _{10}\left[2^{1} 2^{0} \theta^{1}(1-\theta)^{0}\right]$ & 0.5 & 0.0 \\
\hline $7-10$ & $Z=\log _{10}\left[2^{1} 2^{0} \theta^{1}(1-\theta)^{0}\right]$ & 0.5 & 0.0 \\
\hline $8-9$ & $Z=\log _{10}\left[2^{1} 2^{0} \theta^{1}(1-\theta)^{0}\right]$ & 0.5 & 0.0 \\
\hline $8-10$ & $Z=\log _{10}\left[2^{1} 2^{0} \theta^{1}(1-\theta)^{0}\right]$ & 0.5 & 0.0 \\
\hline
\end{tabular}

Table 23. Linkage analysis of family 19.

\begin{tabular}{cccc}
\hline Combination of Markers & LOD & $\theta_{\text {max }}$ & $Z_{\text {max }}^{(19)}$ \\
\hline $1-2$ & $Z=\log _{10}\left[2^{1} 2^{2} \theta^{1}(1-\theta)^{2}\right]$ & 0.33 & 0.07 \\
$1-3$ & $Z=\log _{10}\left[2^{2} 2^{1} \theta^{2}(1-\theta)^{1}\right]$ & 0.5 & 0.0 \\
$1-4$ & $Z=\log _{10}\left[2^{1} 2^{2} \theta^{1}(1-\theta)^{2}\right]$ & 0.33 & 0.07 \\
$1-5$ & $Z=\log _{10}\left[2^{3} 2^{0} \theta^{3}(1-\theta)^{0}\right]$ & 0.5 & 0.0 \\
$1-6$ & $Z=\log _{10}\left[2^{2} 2^{1} \theta^{2}(1-\theta)^{1}\right]$ & 0.5 & 0.0 \\
$1-7$ & $Z=\log _{10}\left[2^{2} 2^{1} \theta^{2}(1-\theta)^{1}\right]$ & 0.5 & 0.0 \\
\hline
\end{tabular}




\section{Continued}

\begin{tabular}{|c|c|c|c|}
\hline $1-9$ & $Z=\log _{10}\left[2^{0} 2^{3} \theta^{0}(1-\theta)^{3}\right]$ & 0.0 & 0.90 \\
\hline $1-10$ & $Z=\log _{10}\left[2^{0} 2^{3} \theta^{0}(1-\theta)^{3}\right]$ & 0.0 & 0.90 \\
\hline $2-3$ & $Z=\log _{10}\left[2^{2} 2^{1} \theta^{2}(1-\theta)^{1}\right]$ & 0.5 & 0.0 \\
\hline $2-4$ & $Z=\log _{10}\left[2^{2} 2^{1} \theta^{2}(1-\theta)^{1}\right]$ & 0.5 & 0.0 \\
\hline $2-5$ & $Z=\log _{10}\left[2^{3} 2^{0} \theta^{3}(1-\theta)^{0}\right]$ & 0.5 & 0.0 \\
\hline $2-6$ & $Z=\log _{10}\left[2^{3} 2^{0} \theta^{3}(1-\theta)^{0}\right]$ & 0.5 & 0.0 \\
\hline $2-7$ & $Z=\log _{10}\left[2^{3} 2^{0} \theta^{3}(1-\theta)^{0}\right]$ & 0.5 & 0.0 \\
\hline $2-8$ & $Z=\log _{10}\left[2^{3} 2^{0} \theta^{3}(1-\theta)^{0}\right]$ & 0.5 & 0.0 \\
\hline $2-9$ & $Z=\log _{10}\left[2^{1} 2^{2} \theta^{1}(1-\theta)^{2}\right]$ & 0.33 & 0.07 \\
\hline $2-10$ & $Z=\log _{10}\left[2^{1} 2^{2} \theta^{1}(1-\theta)^{2}\right]$ & 0.33 & 0.07 \\
\hline $3-4$ & $Z=\log _{10}\left[2^{2} 2^{1} \theta^{2}(1-\theta)^{1}\right]$ & 0.5 & 0.0 \\
\hline $3-5$ & $Z=\log _{10}\left[2^{3} 2^{0} \theta^{3}(1-\theta)^{0}\right]$ & 0.5 & 0.0 \\
\hline $3-6$ & $Z=\log _{10}\left[2^{3} 2^{0} \theta^{3}(1-\theta)^{0}\right]$ & 0.5 & 0.0 \\
\hline $3-7$ & $Z=\log _{10}\left[2^{3} 2^{0} \theta^{3}(1-\theta)^{0}\right]$ & 0.5 & 0.0 \\
\hline $3-8$ & $Z=\log _{10}\left[2^{3} 2^{0} \theta^{3}(1-\theta)^{0}\right]$ & 0.5 & 0.0 \\
\hline $3-9$ & $Z=\log _{10}\left[2^{1} 2^{2} \theta^{1}(1-\theta)^{2}\right]$ & 0.33 & 0.07 \\
\hline $3-10$ & $Z=\log _{10}\left[2^{1} 2^{2} \theta^{1}(1-\theta)^{2}\right]$ & 0.33 & 0.07 \\
\hline $4-5$ & $Z=\log _{10}\left[2^{3} 2^{0} \theta^{3}(1-\theta)^{0}\right]$ & 0.5 & 0.0 \\
\hline $4-6$ & $Z=\log _{10}\left[2^{2} 2^{1} \theta^{2}(1-\theta)^{1}\right]$ & 0.5 & 0.0 \\
\hline $4-7$ & $Z=\log _{10}\left[2^{2} 2^{1} \theta^{2}(1-\theta)^{1}\right]$ & 0.5 & 0.0 \\
\hline $4-8$ & $Z=\log _{10}\left[2^{2} 2^{1} \theta^{2}(1-\theta)^{1}\right]$ & 0.5 & 0.0 \\
\hline $4-9$ & $Z=\log _{10}\left[2^{1} 2^{2} \theta^{1}(1-\theta)^{2}\right]$ & 0.33 & 0.07 \\
\hline $4-10$ & $Z=\log _{10}\left[2^{1} 2^{2} \theta^{1}(1-\theta)^{2}\right]$ & 0.33 & 0.07 \\
\hline $5-6$ & $Z=\log _{10}\left[2^{3} 2^{0} \theta^{3}(1-\theta)^{0}\right]$ & 0.5 & 0.0 \\
\hline $5-7$ & $Z=\log _{10}\left[2^{3} 2^{0} \theta^{3}(1-\theta)^{0}\right]$ & 0.5 & 0.0 \\
\hline $5-8$ & $Z=\log _{10}\left[2^{3} 2^{0} \theta^{3}(1-\theta)^{0}\right]$ & 0.5 & 0.0 \\
\hline $5-9$ & $Z=\log _{10}\left[2^{3} 2^{0} \theta^{3}(1-\theta)^{0}\right]$ & 0.5 & 0.0 \\
\hline $5-10$ & $Z=\log _{10}\left[2^{3} 2^{0} \theta^{3}(1-\theta)^{0}\right]$ & 0.5 & 0.0 \\
\hline $6-7$ & $Z=\log _{10}\left[2^{2} 2^{1} \theta^{2}(1-\theta)^{1}\right]$ & 0.5 & 0.0 \\
\hline $6-8$ & $Z=\log _{10}\left[2^{2} 2^{1} \theta^{2}(1-\theta)^{1}\right]$ & 0.5 & 0.0 \\
\hline $6-9$ & $Z=\log _{10}\left[2^{2} 2^{1} \theta^{2}(1-\theta)^{1}\right]$ & 0.5 & 0.0 \\
\hline
\end{tabular}




\section{Continued}

$\begin{array}{llll}\text { 6- } 10 & Z=\log _{10}\left[2^{2} 2^{1} \theta^{2}(1-\theta)^{1}\right] & 0.5 & 0.0 \\ 7-8 & Z=\log _{10}\left[2^{2} 2^{1} \theta^{2}(1-\theta)^{1}\right] & 0.5 & 0.0 \\ 7-9 & Z=\log _{10}\left[2^{2} 2^{1} \theta^{2}(1-\theta)^{1}\right] & 0.5 & 0.0 \\ 7-10 & Z=\log _{10}\left[2^{2} 2^{1} \theta^{2}(1-\theta)^{1}\right] & 0.5 & 0.0 \\ 8-9 & Z=\log _{10}\left[2^{2} 2^{1} \theta^{2}(1-\theta)^{1}\right] & 0.5 & 0.0 \\ 8-10 & Z=\log _{10}\left[2^{2} 2^{1} \theta^{2}(1-\theta)^{1}\right] & 0.5 & 0.0\end{array}$

